\title{
CONTENTS OF VOLUME 80, 2016
}

\section{Message From the Editor-in-Chief}

1. The Circulation Journal in the World

Hiroaki Shimokawa

2. Editorial Statistics and Best Reviewers Award for 2015

Hiroaki Shimokawa

3. Circulation Journal Awards for the Year 2015

Hiroaki Shimokawa

4. Rapid Publication and Announcement of the 2016 JCS Meeting Activities Hiroaki Shimokawa

5. Announcement of the 2016 JCS Meeting Activities (2)

Hiroaki Shimokawa

6. The 8-Year Journey With the Circulation Journal - 2008 2016 Hiroaki Shimokawa

7. Greetings From the New Editor-in-Chief Toyoaki Murohara

8. Message From the Editor-in-Chief Toyoaki Murohara

9. Message From the Editor-in-Chief Toyoaki Murohara

10. Message From the Editor-in-Chief Toyoaki Murohara

\section{In Memoriam}

1. Henry Krum, PhD 1958-2015

David Cyranoski

Reviews

Reviews (33)

1. Mode of Death After Acute Heart Failure Hospitalization - A Clue to Possible Mechanisms Kishan S. Parikh, G. Michael Felker, Marco Metra

2. Revisiting the Anatomy of the Living Heart Shumpei Mori, Diane E. Spicer, Robert H. Anderson

3. Behavioral Cardiovascular Risk Factors - Effect of Physical Activity and Cardiorespiratory Fitness on Cardiovascular Outcomes -

Rachel M. Spencer, Bettina Heidecker, Peter Ganz

4. The Who, What, Why, When, How and Where of Vasospastic Angina

John F. Beltrame, Filippo Crea, Juan Carlos Kaski, Hisao Ogawa, Peter Ong, Udo Sechtem, Hiroaki Shimokawa,

C. Noel Bairey Merz on behalf of the Coronary Vasomotion Disorders International Study Group (COVADIS)

5. Role of Coronary Microvascular Dysfunction in Takotsubo Cardiomyopathy Cristiana Vitale, Giuseppe MC Rosano, Juan Carlos Kaski

6. Pearls and Pitfalls in Catheter Ablation of Persistent Atrial Fibrillation Li-Wei Lo, Yenn-Jiang Lin, Shih-Lin Chang, Yu-Feng Hu, Fa-Po Chung, Shih-Ann Chen

7. Is Extracorporeal Membrane Oxygenator a New Weapon to Improve Prognosis in Patients With Profound Cardiogenic Shock Undergoing Primary Percutaneous Coronary Intervention?

Pei-Hsun Sung, Chiung-Jen Wu, Hon-Kan Yip

8. Ultra-High-Definition Mapping of Atrial Arrhythmias Sok-Sithikun Bun, Decebal Gabriel Latcu, Tahar Delassi, Mohammed El Jamili, Alaa Al Amoura, Nadir Saoudi

9. Regenerated Endothelium and Its Senescent Response to Aggregating Platelets Paul M. Vanhoutte

10. Antithrombotic Therapy for Secondary Prevention in Patients With Diabetes Mellitus and Coronary Artery Disease Yongwhi Park, Francesco Franchi, Fabiana Rollini, Dominick J Angiolillo

11. Testing in Patients With Stable Coronary Artery Disease - The Debate Continues Udo Sechtem, Heiko Mahrholdt, Peter Ong, Anastasios Athanasiadis, Tim Schäufele 
12. Predicting Atrial Fibrillation and Its Complications Alvaro Alonso, Faye L. Norby

13. Future of the Prevention and Treatment of Coronary Artery Disease Philippe Gabriel Steg, Grégory Ducrocq

14. Catheter Ablation of Idiopathic Ventricular Arrhythmias Arising From the Cardiac Outflow Tracts - Recent Insights and Techniques for the Successful Treatment of Common and Challenging Cases -

Christian-Hendrik Heeger, Kentaro Hayashi, Karl-Heinz Kuck, Feifan Ouyang

15. Implications of Using the Cabrera Sequence for Diagnosing Acute Coronary Syndrome Masami Kosuge, Kazuo Kimura

16. Magnetic Resonance Imaging and Positron Emission Tomography Approaches to Imaging Vascular and Cardiac Inflammation Myriam Amsallem, Toshinobu Saito, Yuko Tada, Rajesh Dash, Michael V. McConnell

17. Redox Regulation of Ischemic Angiogenesis - Another Aspect of Reactive Oxygen Species Yosuke Watanabe, Richard A. Cohen, Reiko Matsui

18. Catecholaminergic Polymorphic Ventricular Tachycardia Krystien V. Lieve, Christian van der Werf, Arthur A. Wilde

19. Mechanical Cardiopulmonary Resuscitation In and On the Way to the Cardiac Catheterization Laboratory Preethi William, Prashant Rao, Uday B. Kanakadandi, Alejandro Asencio, Karl B. Kern

20. Myocardial Fibrosis in Congenital Heart Disease Rahul H. Rathod, Andrew J. Powell, Tal Geva

21. Rho Kinases and Cardiac Remodeling Toru Shimizu, James K. Liao

22. Neurovascular Protective Function of Endothelial Nitric Oxide - Recent Advances Zvonimir S. Katusic, Susan A. Austin

atent Foramen Ovale and Stroke

Yee-Ping Sun, Shunichi Homma

24. Current Trends and Future Perspectives of State-of-the-Art Proteomics Technologies Applied to Cardiovascular Disease Research Sasha A. Singh, Elena Aikawa, Masanori Aikawa

25. Peripartum Cardiomyopathy From a Genetic Perspective Chizuko A. Kamiya, Jun Yoshimatsu, Tomoaki Ikeda

26. Novel Therapies for Heart Failure - Where Do They Stand? Barry Greenberg

27. Optical Coherence Tomography for Online Guidance of Complex Coronary Interventions Kunihiro Shimamura, Giulio Guagliumi

28. Protective Roles of Adipocytokines and Myokines in Cardiovascular Disease Noriyuki Ouchi, Koji Ohashi, Rei Shibata, Toyoaki Murohara

9. Heart Development, Diseases, and Regeneration - New Approaches From Innervation, Fibroblasts, and Reprogramming Masaki Ieda

30. Macrophage Apoptosis and Efferocytosis in the Pathogenesis of Atherosclerosis MacRae F. Linton, Vladimir R. Babaev, Jiansheng Huang, Edward F. Linton, Huan Tao, Patricia G. Yancey

\section{Defining the Cardiac Fibroblast
Malina J. Ivey, Michelle D. Tallquist \\ 31. Defining the Cardiac Fibroblast
Malina J. Ivey, Michelle D. Tallquist} Genjiro Kimura

33. Traditional Cardiovascular Risk Factors for Incident Atrial Fibrillation Yoshihiro Kokubo, Chisa Matsumoto

Focus Reviews on Coronary Artery Disease (2)

34. Calpains and Coronary Vascular Disease Brittany A. Potz, Ashraf A. Sabe, M. Ruhul Abid, Frank W. Sellke

35. The What, When, Who, Why, How and Where of Myocardial Infarction With Non-Obstructive Coronary Arteries (MINOCA) Sivabaskari Pasupathy, Rosanna Tavella, John F. Beltrame

Focus Reviews on Heart Failure (2)

36. From Left Ventricular Hypertrophy to Dysfunction and Failure Davide Lazzeroni, Ornella Rimoldi, Paolo G Camici

37. Optimal Use of Beta-Blockers for Congestive Heart Failure Hae-Young Lee, Sang Hong Baek

Focus Reviews on the Hippo Pathway (2)

38. Hippo Signaling in the Heart - Non-Canonical Pathways Impact Growth, Survival and Function Dominic P. Del Re 
39. Regulation of Myocardial Cell Growth and Death by the Hippo Pathway

Shohei Ikeda, Junichi Sadoshima

\section{Report of Heart Transplantation in Japan}

1. The Registry Report of Heart Transplantation in Japan (1999-2014)

Takeshi Nakatani, Norihide Fukushima, Minoru Ono, Yoshikatsu Saiki, Hikaru Matsuda, Shinichi Nunoda, Yoshiki Sawa,

Mitsuaki Isobe

\section{Congress Reports}

2015 AHA Report

1. Report of the American Heart Association (AHA) Scientific Sessions 2015, Orlando Yoshiyasu Aizawa, Mai Kimura, Takashi Kohno, Jun Fujita, Keiichi Fukuda

2016 ACC Report

2. Report of the American College of Cardiology (ACC) Scientific Sessions 2016, Chicago Toshiaki Mano, Kazuhiro Yamamoto

\section{JCS Report}

3. Overview of the $80^{\text {th }}$ Annual Scientific Meeting of the Japanese Circulation Society - The Past, Present and Future of Cardiovascular Medicine in Japan - - The $5^{\text {th }}$ Anniversary of the Great East Japan Earthquake -

Jun Takahashi, Kimio Satoh, Koji Fukuda, Koichiro Sugimura, Yasuharu Matsumoto, Makoto Nakano, Ryuji Tsuburaya, Tatsuo Aoki, Kiyotaka Hao, Kensuke Nishimiya, Kenta Ito, Yasuhiko Sakata, Hiroaki Shimokawa

\section{ESC Report}

4. European Society of Cardiology (ESC) Annual Congress Report From Rome 2016 Hiroya Mizuno, Tomohito Otani, Yasushi Sakata

\section{Editorials}

1. Usefulness of Tissue Doppler Imaging-Derived Atrial Conduction Time for Prediction of Atrial Fibrillation Toshinori Yuasa, Yutaka Imoto

2. Respiratory Intervention in Patients With Chronic Heart Failure Takashi Koyama, Hiroyuki Watanabe, Hiroshi Ito

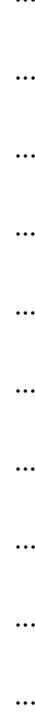

3. Is New-Onset Atrial Fibrillation Bad News for Patients With Heart Failure? Moritake Iguchi, Masaharu Akao

4. Epicardial Adipose Tissue as a Predictor of Plaque Vulnerability in Patients With Mild Chronic Kidney Disease Atsushi Nohara

5. Is It Possible to Predict the Onset of "Heart Failure" in Hypertrophic Cardiomyopathy? Keiko Yamauchi-Takihara

6. "Treat-and-Repair" Strategy for Atrial Septal Defect and Associated Pulmonary Arterial Hypertension Atsushi Yao

7. The Roles of Classic and Current Prognostic Factors in Pulmonary Hypertension Due to Left Heart Disease Akihiro Hirashiki, Takahisa Kondo, Toyoaki Murohara

8. Do the Components of $\mathrm{CHA}_{2} \mathrm{DS}_{2}-\mathrm{VAsc}$ Score Affect Stroke Severity and Outcome? Takeshi Hayashi

9. Taking Control of the Time Bomb in Abdominal Aortic Aneurysm Hiroki Aoki

10. Triple Antithrombotic Therapy - Always One Too Many? Hidehira Fukaya, Junya Ako

11. How Should We Treat Myocardial Infarction Patients With Multivessel Disease? - Staged Revascularization? Or Stent Them All Acutely? -

Kenichi Tsujita, Kenshi Yamanaga, Koichi Kaikita, Seiji Hokimoto, Hisao Ogawa

12. Eicosapentaenoic Acid Added to Strong Statin Therapy Shiro Nakahara, Takaaki Komatsu, Isao Taguchi

13. Low-Density Lipoprotein Cholesterol Level and Statin Therapy in Patients With Acute Myocardial Infarction (Cholesterol Paradox)

Tsuyoshi Nozue

14. Ischemic Burden Size, Left Ventricular Function or Both for Better Revascularization Outcomes? Tomoaki Nakata, Koki Abe

15. Pentraxin 3 - A Link Between Obesity, Inflammation and Vascular Disease? Francesco Violi, Daniele Pastori 
16. Lipoprotein(a): Revisiting a Next Therapeutic Target Kouji Kajinami

17. Atrial Fibrillation and Heart Failure - Identification of Patients at Risk David E. Krummen

18. Coronary Angioscopy for the Evaluation of Vessel Response After Drug-Eluting Stent Implantation Masahiko Shibuya, Masaharu Ishihara

19. Impact of Insulin Resistance on Clinical Outcomes After Implantation of Drug-Eluting Stents Masaaki Miyata

20. Here Comes a Novel Heart-Rate Reducing Therapy in Heart Failure Treatment Tetsu Watanabe

21. Factors Associated With Metabolic Syndrome That Affect Prognosis in Heart Failure Patients Susumu Adachi

22. Clinical Features of Long QT Syndrome in Children Naokata Sumitomo

23. Patent Ductus Arteriosus - A Complex Problem in Need of a Solid Conceptual Foundation Carl H. Backes, Charles V. Smith

24. "Get With the Guideline" Treatment With Statin Hayato Matsushima, Naohisa Hosomi

25. Innovative Edoxaban Overcomes Undesirable Characteristics of Warfarin in East Asian Patients Masahiro Yasaka

26. Silent Cerebral Embolism After Catheter Ablation for Atrial Fibrillation - Unresolved Issue or Too Much Concern? Teiichi Yamane

27. Implantation Site Does Matter for Defibrillation Threshold Yasuhiro Yokoyama

28. Developmental Origins of Cardiovascular Dysfunction - Doomed From Birth? Michael D. Thompson, Aaron J. Trask

29. High Fibrinogen Plus Low Plasminogen Levels Predict Poor Post-Surgical Survival in Patients With Chronic Thromboembolic Pulmonary Hypertension Manabu Horii

30. Systemic Abnormalities Derived From Chronic Thromboembolic Pulmonary Hypertension and Their Improvement by Balloon Pulmonary Angioplasty

Toru Satoh

31. Knowing the Risks of the Vessels From the Vessels Tetsuya Matoba

32. Percutaneous Left Atrial Appendage Closure for Non-Valvular Atrial Fibrillation Hidehiko Hara

33. Bioresorbable Scaffold - Taking the Edge Off? Atsushi Tanaka

34. Cardiopulmonary Resuscitation in a Super-Aging Society - Is There an Age Limit for Cardiopulmonary Resuscitation? Yoshio Tahara

35. Estimating Left Ventricular Relaxation and Filling Pressure Using Speckle Tracking Strain

Hidekazu Tanaka
36. Lower Radiation Exposure Needs Lower Heart Rate Hideya Yamamoto, Yasuki Kihara

37. The Challenges of Transfer and Transition in Congenital Heart Disease Christopher P. Learn, John Lynn Jefferies

38. Dilated Cardiomyopathy in Children With Isolated Congenital Complete Atrioventricular Block Hitoshi Horigome

39. Some Methodological Issues and Future of Risk Prediction Studies in Cardiology Hideki Origasa

40. Encouraging Results of Renal Denervation in Resistant Hypertension Patients With Obstructive Sleep Apnea Hiroo Kumagai, Naoki Oshima

41. Shining the Light on Calcium in the Catheterization Lab Stephen J. Nicholls, Yu Kataoka, Peter J. Psaltis

42. Noninvasive Vascular Function Tests - Long Journey for Predicting Cardiovascular Events Kenya Kusunose, Hirotsugu Yamada

43. Current Treatment Strategy for Spontaneous Isolated Dissection of the Superior Mesenteric Artery Hitoshi Ogino

44. Beneficial Therapeutic Effects of Balloon Pulmonary Angioplasty on Biventricular Function in Patients With Chronic Thromboembolic Pulmonary Hypertension Norikazu Yamada 
45. Regional Differences in Warfarin Therapy Among Japanese Patients With Atrial Fibrillation Yasushi Miyauchi

46. Specific Problems With Accidental Deaths in Japan - Cardiac Arrest From Hot Baths and Foreign Body Airway Obstruction in Elders -

Hiroyuki Hanada

47. Insomnia in Heart Failure Miyuki Tsuchihashi-Makaya, Shiho Matsuoka

48. Effect of Aging on Fractional Flow Reserve - Hyperemic Index Fractional Flow Reserve May Not Be Sufficient to Reveal the Whole Picture of Coronary Circulation Hitoshi Matsuo

49. The Paradox of Smoking and Clopidogrel Effect - Dr Jekyll or Mr Hyde? Fabiana Rollini, Francesco Franchi

50. There Is Much to Be Gained by Discarding Preconceived Notions Jun Takahashi

51. Transient Atrial Fibrillation During Acute Myocardial Infarction Is a Predictor of Poor Outcomes Masatake Fukunami

52. Systemic Inflammation Is a Key Factor for Mortality Risk Stratification in Chronic Kidney Disease Patients With Coronary Artery Calcification Fumiki Yoshihara

53. Hope or Hype - Does Cryoballoon Simplify Atrial Fibrillation Ablation Technique or Just Another Costly Toy? Li-Wei Lo, Shih-Ann Chen

54. Late Phase II Cardiac Rehabilitation, Which Is Adding Life to Years and Years to Life, Is Still Underused in Japan Masahiro Kohzuki

55. Redefinition of Prehospital Area as Critical Target for ST-Elevation Myocardial Infarction Care - "Time Is Myocardium" Hideo Fujita

56. Is the Brain the Alternative Therapeutic Target for Heart Failure?

Tetsu Watanabe

57. Fractional Flow Reserve, Coronary Pressure Wires, and Drift Nico HJ Pijls, Bernard De Bruyne

58. Trunk-to-Leg Fat Ratio - An Emerging Early Marker of Childhood Adiposity, and Future Cardiometabolic Risks Ichiro Kishimoto

59. Hyperuricemia Plays Pivotal Role in Progression of Kidney Disease Ichiro Hisatome, Masanari Kuwabara

60. An Ideal Time to Solve a Clinical Dilemma in the Golden Age of Aortic Stenosis Therapy Yoshihiro Seo, Kazutaka Aonuma

61. Long-Term Prognosis of Catecholaminergic Polymorphic Ventricular Tachycardia Patients With Ryanodine Receptor (RYR2) Mutations Masahiro Yamazoe, Tetsushi Furukawa

62. Angioscopic Assessment of Stent Stability and Neointimal Coverage After Implantation of 2nd-Generation Drug-Eluting Stents - Comparison With Bare-Mental Stents and 1st-Generation Drug-Eluting Stents Shigeki Kimura

63. Importance of Out-of-Clinic Blood Pressure Measurement for Device-Based Hypertensive Therapy Satoshi Hoshide, Kazuomi Kario

64. Left Ventricular Assist Devices and Small Body Surface Area - A Clinical Concern? Michael Ibrahim, Arman Kilic, Pavan Atluri

65. Dawn of a New Era - The Far Lower, the Far Better Low-Density Lipoprotein Cholesterol Story in Japan Tatsuro Ishida

66. Interaction Between Brain and Heart Satoshi Kurisu, Yasuki Kihara

67. What Scoring System Should We Use to Assess Bleeding Risk in Atrial Fibrillation? Yasuo Okumura

68. Aging - The Great Impact on Coagulation State -

Naohiko Takahashi

69. Proper Salt Intake During Pregnancy Katsuyuki Ando

70. Cardiovascular Remodeling in the Small for Gestational Age Infant - Implications and Future Directions Tyler A. Fick, Carl H. Backes

71. "Hamstring Leaflet" Now Demonstrated to Be a Determinant of Functional Mitral Regurgitation in Patients With Atrial Fibrillation Tetsuhiro Yamano, Keizo Furukawa, Satoaki Matoba

72. Enhanced Vasa Vasorum Formation at Spasm Site - Coincident Plexus or External Pathogenic Routes? Atsushi Tanaka, Kenei Shimada 
73. Current Optimal Anticoagulation Regimen of Rivaroxaban in Atrial Fibrillation Catheter Ablation Yu-ki Iwasaki, Wataru Shimizu

74. Diagnosis Procedure Combination Database Would Develop Nationwide Clinical Research in Japan Koshi Nakamura

75. Calcium-Channel Blockers as Antidementia Drugs Masaki Mogi, Masatsugu Horiuchi

76. Trends in the Impact of Diabetes on the Development of Cardiovascular Diseases Kyoung Hwa Ha, Dae Jung Kim

77. Rare Variants in ANK2 Associated With Various Inherited Arrhythmia Syndromes Hiroshi Watanabe, Tohru Minamino

78. Obesity and Overweight in Asian People Akiko Goda, Tohru Masuyama

79. Myocardial Tissue Characterization of Left Ventricular Reverse Remodeling in Ischemic Cardiomyopathy Yoji Nagata, Tetsuo Konno, Kenshi Hayashi, Masa-aki Kawashiri

80. The Conundrum of Platelet P2Y12 Inhibition in ST-Segment Elevation Myocardial Infarction Fabiana Rollini, Francesco Franchi

81. Impact of Legislation for Smoking Cessation and the Tokyo Olympic and Paralympic Games 2020 Akinori Fujikake, Takaaki Komatsu, Shiro Nakahara, Isao Taguchi

\section{Late Breaking Clinical Trials (JCS 2016)}

1. Waon Therapy for Managing Chronic Heart Failure - Results From a Multicenter Prospective Randomized WAON-CHF Study -

Chuwa Tei, Teruhiko Imamura, Koichiro Kinugawa, Teruo Inoue, Tohru Masuyama, Hiroshi Inoue, Hirofumi Noike, Toshihiro Muramatsu, Yasuchika Takeishi, Keijiro Saku, Kazumasa Harada, Hiroyuki Daida, Youichi Kobayashi, Nobuhisa Hagiwara, Masatoshi Nagayama, Shinichi Momomura, Kazuya Yonezawa, Hiroshi Ito, Satoshi Gojo, Makoto Akaishi, Masaaki Miyata, Mitsuru Ohishi, WAON-CHF Study Investigators

2. Efficacy and Safety of Inhaled Iloprost in Japanese Patients With Pulmonary Arterial Hypertension - Insights From the IBUKI and AIR Studies -

Tsutomu Saji, Masafumi Myoishi, Koichiro Sugimura, Nobuhiro Tahara, Yutaka Takeda, Keiichi Fukuda, Horst Olschewski, Yoshimi Matsuda, Sylvia Nikkho, Toru Satoh

3. A Randomized Controlled Study of Finerenone vs. Eplerenone in Japanese Patients With Worsening Chronic Heart Failure and Diabetes and/or Chronic Kidney Disease

Naoki Sato, Masayoshi Ajioka, Takahisa Yamada, Masaharu Kato, Masafumi Myoishi, Takashi Yamada, So-Young Kim, Christina Nowack, Peter Kolkhof, Tsuyoshi Shiga on behalf of the ARTS-HF Japan study group

\section{Late Breaking Cohort Studies (JCS 2016)}

1. Beneficial Effect of Non-Vitamin K Antagonist Oral Anticoagulants in Patients With Nonvalvular Atrial Fibrillation - Results of the J-RHYTHM Registry 2 -

Eitaro Kodani, Hirotsugu Atarashi, Hiroshi Inoue, Ken Okumura, Takeshi Yamashita, Hideki Origasa on behalf of the J-RHYTHM Registry Investigators

2. Characteristics and Predictors of Mortality in Patients With Cardiovascular Shock in Japan - Results From the Japanese Circulation Society Cardiovascular Shock Registry -

Yasushi Ueki, Masahiro Mohri, Tetsuya Matoba, Yasuyuki Tsujita, Masao Yamasaki, Eizo Tachibana, Naohiro Yonemoto, Ken Nagao

\section{Original Articles}

Aortic Disease (2)

1. Inverse Correlation Between Calcium Accumulation and the Expansion Rate of Abdominal Aortic Aneurysms Atsuko Nakayama, Hiroyuki Morita, Naoto Hayashi, Yukihiro Nomura, Katsuyuki Hoshina, Kunihiro Shigematsu, Hiroshi Ohtsu, Tetsuro Miyata, Issei Komuro

2. Hemodynamic Analysis of Endoleaks After Endovascular Abdominal Aortic Aneurysm Repair by Using 4-Dimensional Flow-Sensitive Magnetic Resonance Imaging Mayu Sakata, Yasuo Takehara, Kazuto Katahashi, Masaki Sano, Kazunori Inuzuka, Naoto Yamamoto, Masataka Sugiyama, Harumi Sakahara, Tetsuya Wakayama, Marcus T. Alley, Hiroyuki Konno, Naoki Unno

Arrhythmia/Electrophysiology (33)

3. Effect of Carbenoxolone on Arrhythmogenesis in Rat Ventricular Muscle Masahito Miura, Tsuyoshi Nagano, Naomi Murai, Yuhto Taguchi, Tetsuya Handoh, Minami Satoh, Satoshi Miyata, Lawson Miller, Chiyohiko Shindoh, Bruno D. Stuyvers 
4. Efficacy and Myocardial Injury With Subcutaneous Implantable Cardioverter Defibrillators - Computer Simulation of Defibrillation Shock Conduction -

Mahito Noro, Xin Zhu, Yoshinari Enomoto, Masako Asami, Rina Ishii, Yasutake Toyoda, Naohiko Sahara, Takahito Takagi, Yuriko Narabayasi, Hikari Hashimoto, Naoshi Ito, Shingo Kujime, Yasuhiro Oikawa, Hiroyuki Tatsunami, Tsuyoshi Sakai, Keijirou Nakamura, Takao Sakata, Kaoru Sugi

5. Pronounced Shortening of QT Interval With Mexiletine Infusion Test in Patients With Type 3 Congenital Long QT Syndrome

Moritoshi Funasako, Takeshi Aiba, Kohei Ishibashi, Ikutaro Nakajima, Koji Miyamoto, Yuko Inoue, Hideo Okamura, Takashi Noda, Shiro Kamakura, Toshihisa Anzai, Teruo Noguchi, Satoshi Yasuda, Yoshihiro Miyamoto, Kengo Fukushima Kusano, Hisao Ogawa, Wataru Shimizu

6. Early Recurrence After Pulmonary Vein Isolation of Paroxysmal Atrial Fibrillation With Different Ablation Technologies - Prospective Comparison of Radiofrequency vs. Second-Generation Cryoballoon Ablation -

Shinsuke Miyazaki, Akio Kuroi, Hitoshi Hachiya, Hiroaki Nakamura, Hiroshi Taniguchi, Noboru Ichihara, Takamitsu Takagi, Jin Iwasawa, Yoshito Iesaka

7. Effects of Triple Therapy in Patients With Non-Valvular Atrial Fibrillation Undergoing Percutaneous Coronary Intervention Regarding Thromboembolic Risk Stratification

Antonia Sambola, Maria Mutuberría, Bruno García del Blanco, Albert Alonso, José A. Barrabés, Fernando Alfonso, Héctor Bueno, Angel Cequier, Javier Zueco, Oriol Rodríguez-Leor, Eduard Bosch, Pilar Tornos, David García-Dorado

8. Long-Term Predictors of Thromboembolic Events in Nonvalvular Atrial Fibrillation Patients Undergoing Electrical Cardioversion

Amaya García-Fernández, Francisco Marín, Vanessa Roldán, José M Gómez-Sansano, Diana Hernández-Romero, Mariano Valdés, Juan G Martinez-Martinez, Francisco Sogorb-Garri, Gregory YH Lip

9. Outcome of Patients Treated by Cardiac Resynchronization Therapy Using a Quadripolar Left Ventricular Lead Gianluigi Bencardino, Antonio Di Monaco, Eleonora Russo, Cristian Colizzi, Francesco Perna, Gemma Pelargonio, Maria Lucia Narducci, Francesca Augusta Gabrielli, Gaetano Antonio Lanza, Antonio Giuseppe Rebuzzi, Filippo Crea

10. Clinical and Echocardiographic Factors Associated With New-Onset Atrial Fibrillation in Heart Failure - Subanalysis of the WARCEF Trial -

Tomoko S. Kato, Marco R. Di Tullio, Min Qian, Mengfei Wu, John L.P. Thompson, Douglas L. Mann, Ralph L. Sacco, Patrick M. Pullicino, Ronald S. Freudenberger, John R. Teerlink, Susan Graham, Gregory Y.H. Lip, Bruce Levin, Jay P. Mohr, Arthur J. Labovitz, Conrado J. Estol, Dirk J. Lok, Piotr Ponikowski, Stefan D. Anker, Shunichi Homma for the WARCEF Investigators

11. Elevated Red Blood Cell Distribution Width Predicts Recurrence After Catheter Ablation for Atrial Fibrillation in Patients With Heart Failure - Comparison With Non-Heart Failure Patients -

Satoshi Yanagisawa, Yasuya Inden, Hiroyuki Kato, Aya Miyoshi, Yoshiaki Mizutani, Tadahiro Ito, Yosuke Kamikubo, Yasunori Kanzaki, Makoto Hirai, Toyoaki Murohara

12. Nine-Year Trend of Anticoagulation Use, Thromboembolic Events, and Major Bleeding in Patients With Non-Valvular Atrial Fibrillation - Shinken Database Analysis -

Shinya Suzuki, Takayuki Otsuka, Koichi Sagara, Hiroaki Semba, Hiroto Kano, Shunsuke Matsuno, Hideaki Takai, Yuko Kato, Tokuhisa Uejima, Yuji Oikawa, Kazuyuki Nagashima, Hajime Kirigaya, Takashi Kunihara, Junji Yajima, Hitoshi Sawada, Tadanori Aizawa, Takeshi Yamashita

13. Edoxaban vs. Warfarin in East Asian Patients With Atrial Fibrillation - An ENGAGE AF-TIMI 48 Subanalysis -

Takeshi Yamashita, Yukihiro Koretsune, Yuejin Yang, Shih-Ann Chen, Namsik Chung, Yuichi J. Shimada, Tetsuya Kimura, Koichi Miyazaki, Kenji Abe, Michele Mercuri, Christian T. Ruff, Robert P. Giugliano

14. Silent Cerebral Ischemic Lesions After Catheter Ablation of Atrial Fibrillation in Patients on 5 Types of Periprocedural Oral Anticoagulation - Predictors of Diffusion-Weighted Imaging-Positive Lesions and Follow-up Magnetic Resonance Imaging -

Kohki Nakamura, Shigeto Naito, Takehito Sasaki, Kentaro Minami, Yutaka Take, Eri Goto, Satoru Shimizu, Yoshiaki Yamaguchi, Naoko Suzuki, Toshiaki Yano, Michiharu Senga, Koji Kumagai, Kenichi Kaseno, Nobusada Funabashi, Shigeru Oshima

15. Decreased Defibrillation Threshold and Minimized Myocardial Damage With Left Axilla Implantable Cardioverter Defibrillator Implantation

Mahito Noro, Xin Zhu, Yoshinari Enomoto, Yasuhiro Oikawa, Hiroyuki Tatsunami, Rina Ishii, Yasutake Toyoda, Masako Asami, Naohiko Sahara, Takahito Takagi, Yuriko Narabayashi, Hikari Hashimoto, Naoshi Ito, Shingo Kujime, Tsuyoshi Sakai, Keijirou Nakamura, Takao Sakata, Haruhiko Abe, Kaoru Sugi

16. Impact of Catheter Ablation for Paroxysmal Atrial Fibrillation in Patients With Sick Sinus Syndrome - Important Role of Non-Pulmonary Vein Foci -

Kentaro Hayashi, Masato Fukunaga, Kyohei Yamaji, Yoshimori An, Michio Nagashima, Kenichi Hiroshima, Masatsugu Ohe, Yu Makihara, Kennosuke Yamashita, Kenji Ando, Masashi Iwabuchi, Masahiko Goya

17. Left Atrial Appendage Occlusion in Non-Valvular Atrial Fibrillation in a Korean Multi-Center Registry Jung-Sun Kim, Hancheol Lee, Yongsung Suh, Hui-Nam Pak, Geu-Ru Hong, Chi Young Shim, Cheol-Woong Yu, Hyun-Jong Lee, Woong-Chol Kang, Eun-Seok Shin, Rak-Kyeong Choi, Saibal Kar, Jai-Wun Park, Do-Sun Lim, Yangsoo Jang 
18. Right Parasternal Lead Placement Increases Eligibility for Subcutaneous Implantable Cardioverter Defibrillator Therapy in Adults With Congenital Heart Disease

Hideo Okamura, Christopher J. McLeod, Christopher V. DeSimone, Tracy L. Webster, Crystal R. Bonnichsen, Martha Grogan, Sabrina D. Phillips, Heidi M. Connolly, Naser M. Ammash, Carole A. Warnes, Paul A. Friedman

19. Beat-to-Beat Variability in Preload Unmasks Latent Risk of Torsade de Pointes in Anesthetized Chronic Atrioventricular Block Dogs

Thom RG Stams, Peter Oosterhoff, Atty Heijdel, Albert Dunnink, Jet DM Beekman, Roel van der Nagel, Harold VM van Rijen, Marcel AG van der Heyden, Marc A Vos

20. Atrial Fibrillation-Mediated Upregulation of miR-30d Regulates Myocardial Electrical Remodeling of the G-ProteinGated K+ Channel, IK.ACh

Masaki Morishima, Eriko Iwata, Chisato Nakada, Yoshiyuki Tsukamoto, Hiroki Takanari, Shinji Miyamoto, Masatsugu Moriyama, Katsushige Ono

21. Clinical Impact of Ventricular Tachycardia and/or Fibrillation During the Acute Phase of Acute Myocardial Infarction on In-Hospital and 5-Year Mortality Rates in the Percutaneous Coronary Intervention Era

Masaharu Masuda, Daisaku Nakatani, Shungo Hikoso, Shinichiro Suna, Masaya Usami, Sen Matsumoto, Tetsuhisa Kitamura, Hitoshi Minamiguchi, Yuji Okuyama, Masaaki Uematsu, Takahisa Yamada, Katsuomi Iwakura, Toshimitsu Hamasaki, Yasuhiko Sakata, Hiroshi Sato, Shinsuke Nanto, Masatsugu Hori, Issei Komuro, Yasushi Sakata on behalf of the OACIS investigators

22. Regional Differences in Frequency of Warfarin Therapy and Thromboembolism in Japanese Patients With Non-Valvular Atrial Fibrillation - Analysis of the J-RHYTHM Registry -

Hiroshi Inoue, Hirotsugu Atarashi, Eitaro Kodani, Ken Okumura, Takeshi Yamashita, Hideki Origasa, Masayuki Sakurai, Yuichiro Kawamura, Isao Kubota, Kazuo Matsumoto, Yoshiaki Kaneko, Satoshi Ogawa, Yoshifusa Aizawa, Masaomi Chinushi, Itsuo Kodama, Eiichi Watanabe, Yukihiro Koretsune, Yuji Okuyama, Akihiko Shimizu, Osamu Igawa, Shigenobu Bando, Masahiko Fukatani, Tetsunori Saikawa, Akiko Chishaki on behalf of the J-RHYTHM Registry Investigators

23. Pericardial Fat Is Associated With the Risk of Ventricular Arrhythmia in Asian Patients Weng-Chio Tam, Yung-Kuo Lin, Wing-Pong Chan, Jen-Hung Huang, Ming-Hsiung Hsieh, Shih-Ann Chen, Yi-Jen Chen

24. Activation Pattern of the Polymorphic Ventricular Tachycardia and Ventricular Fibrillation on Body Surface Mapping in Patients With Brugada Syndrome

Akira Ueoka, Hiroshi Morita, Atsuyuki Watanabe, Koji Nakagawa, Nobuhiro Nishii, Satoshi Nagase, Tohru Ohe, Hiroshi Ito

25. Safety and Efficacy of Cryoballoon Ablation for Paroxysmal Atrial Fibrillation in Japan - Results From the Japanese Prospective Post-Market Surveillance Study -

Ken Okumura, Kazuo Matsumoto, Yoshinori Kobayashi, Akihiko Nogami, Robert B Hokanson, Fred Kueffer for the CRYO-Japan PMS Study Investigators

26. Catecholaminergic Polymorphic Ventricular Tachycardia (CPVT) Associated With Ryanodine Receptor (RyR2) Gene Mutations - Long-Term Prognosis After Initiation of Medical Treatment -

Hiro Kawata, Seiko Ohno, Takeshi Aiba, Heima Sakaguchi, Aya Miyazaki, Naokata Sumitomo, Tsukasa Kamakura, Ikutaro Nakajima, Yuko Y Inoue, Koji Miyamoto, Hideo Okamura, Takashi Noda, Kengo Kusano, Shiro Kamakura, Yoshihiro Miyamoto, Isao Shiraishi, Minoru Horie, Wataru Shimizu

27. Is the ORBIT Bleeding Risk Score Superior to the HAS-BLED Score in Anticoagulated Atrial Fibrillation Patients? María Asunción Esteve-Pastor, Amaya García-Fernández, Manuel Macías, Francisco Sogorb, Mariano Valdés, Vanessa Roldán, Javier Muñiz, Lina Badimon, Inmaculada Roldán, Vicente Bertomeu-Martínez, Ángel Cequier, Gregory Y.H. Lip, Manuel Anguita, Francisco Marín on behalf of FANTASIIA Investigators

28. Prevalence and Clinical Impact of Early Repolarization Pattern and QRS-Fragmentation in High-Risk Patients With Brugada Syndrome

Giulio Conte, Carlo de Asmundis, Juan Sieira, Giuseppe Ciconte, Giacomo Di Giovanni, Gian-Battista Chierchia, Ruben Casado-Arroyo, Giannis Baltogiannis, Erwin Ströker, Ghazala Irfan, Gudrun Pappaert, Angelo Auricchio, Pedro Brugada

29. Clinical Significance of Peripheral Endothelial Function for Left Atrial Blood Stagnation in Nonvalvular Atrial Fibrillation Patients With Low-to-Intermediate Stroke Risk

Akira Fujii, Katsuji Inoue, Takayuki Nagai, Teruyoshi Uetani, Kazuhisa Nishimura, Jun Suzuki, Jun-ichi Funada, Takafumi Okura, Jitsuo Higaki, Akiyoshi Ogimoto

30. Efficacy and Safety of Rivaroxaban and Warfarin in the Perioperative Period of Catheter Ablation for Atrial Fibrillation - Outcome Analysis From a Prospective Multicenter Registry Study in Japan -

Ken Okumura, Kazutaka Aonuma, Koichiro Kumagai, Kenzo Hirao, Koichi Inoue, Masaomi Kimura, Yasushi Miyauchi, Eiki Tsushima for the JACRE Investigators

31. Pacing From the Right Ventricular Septum and Development of New Atrial Fibrillation in Paced Patients With Atrioventricular Block and Preserved Left Ventricular Function Katsuhide Hayashi, Ritsuko Kohno, Yoshihisa Fujino, Masao Takahashi, Yasushi Oginosawa, Hisaharu Ohe, Tetsu Miyamoto, Shota Fukuda, Masaru Araki, Shinjo Sonoda, Yutaka Otsuji, Haruhiko Abe

32. Ventricular Fibrillation in a General Population - A National Database Study Wei-Chieh Tseng, Mei-Hwan Wu, Hui-Chi Chen, Feng-Yu Kao, San-Kuei Huang 
33. Phenotypic Variability of ANK2 Mutations in Patients With Inherited Primary Arrhythmia Syndromes Mari Ichikawa, Takeshi Aiba, Seiko Ohno, Daichi Shigemizu, Junichi Ozawa, Keiko Sonoda, Megumi Fukuyama, Hideki Itoh, Yoshihiro Miyamoto, Tatsuhiko Tsunoda, Takeru Makiyama, Toshihiro Tanaka, Wataru Shimizu, Minoru Horie

34. M3 Muscarinic Receptor Signaling Stabilizes a Novel Mutant Human Ether-a-Go-Go-Related Gene Channel Protein via Phosphorylation of Heat Shock Factor 1 in Transfected Cells

Endang Mahati, Peili Li, Yasutaka Kurata, Nani Maharani, Nobuhito Ikeda, Shinji Sakata, Kazuyoshi Ogura, Junichiro Miake, Takeshi Aiba, Wataru Shimizu, Naoe Nakasone, Haruaki Ninomiya, Katsumi Higaki, Kazuhiro Yamamoto, Akira Nakai, Yasuaki Shirayoshi, Ichiro Hisatome

35. Uric Acid Level and Prevalence of Atrial Fibrillation in a Japanese General Population of 285,882 Shin Kawasoe, Takuro Kubozono, Shiro Yoshifuku, Satoko Ojima, Naoya Oketani, Masaaki Miyata, Hironori Miyahara, Shigeho Maenohara, Mitsuru Ohishi

Cardiovascular Intervention (13)

36. Temporal Trends in Clinical Outcome After Percutaneous Coronary Intervention 1984-2010 - Report From the Juntendo PCI Registry -

Ryo Naito, Katsumi Miyauchi, Hirokazu Konishi, Shuta Tsuboi, Manabu Ogita, Tomotaka Dohi, Takatoshi Kasai, Hiroshi Tamura, Shinya Okazaki, Kikuo Isoda, Hiroyuki Daida

37. Clinical Outcomes of Drug-Eluting Stents vs. Bare-Metal Stents in Acute Myocardial Infarction Patients Under Dialysis - A Nationwide Cohort Study -

Dong-Yi Chen, Chun-Tai Mao, Ming-Lung Tsai, Ming-Jer Hsieh, Yu-Sheng Lin, Wen-Jin Cherng, Ming-Shien Wen, Chao-Hung Wang, I-Chang Hsieh, Ming-Jui Hung, Chun-Chi Chen, Tien-Hsing Chen

38. Culprit Vessel-Only vs. Staged Multivessel Percutaneous Coronary Intervention Strategies in Patients With Multivessel Coronary Artery Disease Undergoing Primary Percutaneous Coronary Intervention for ST-Segment Elevation Myocardial Infarction

Toshiaki Toyota, Hiroki Shiomi, Tomohiko Taniguchi, Takeshi Morimoto, Yutaka Furukawa, Yoshihisa Nakagawa, Minoru Horie, Takeshi Kimura on behalf of the CREDO-Kyoto AMI Registry Investigators

39. The Leipzig Prospective Drug-Eluting Balloon-Registry - Outcome of 484 Consecutive Patients Treated for Coronary In-Stent Restenosis and De Novo Lesions Using Paclitaxel-Coated Balloons -

Madlen Uhlemann, Sven Möbius-Winkler, Jennifer Adam, Sandra Erbs, Norman Mangner, Marcus Sandri, Enno Boudriot, Michael Woinke, Gerhard C. Schuler, Axel Linke

40. Angioscopic Comparison of Resolute and Endeavor Zotarolimus-Eluting Stents

Yuji Nishimoto, Koshi Matsuo, Yasunori Ueda, Ryuta Sugihara, Akio Hirata, Ayaka Murakami, Kazunori Kashiwase, Yoshiharu Higuchi, Yoshio Yasumura

41. Insulin Resistance as a Predictor of the Late Catch-up Phenomenon After Drug-Eluting Stent Implantation Takaaki Komatsu, Sachiko Komatsu, Hidehiko Nakamura, Takanori Kuroyanagi, Akinori Fujikake, Itaru Hisauchi, Masashi Sakuma, Shiro Nakahara, Yoshihiko Sakai, Isao Taguchi

42. Impact of Culprit Plaque and Atherothrombotic Components on Incomplete Stent Apposition in Patients With STElevation Myocardial Infarction Treated With Everolimus-Eluting Stents - An OCTAVIA Substudy -

Chiara Bernelli, Kunihiro Shimamura, Kenichi Komukai, Davide Capodanno, Francesco Saia, Roberto Garbo, Francesco Burzotta, Vasile Sirbu, Micol Coccato, Gianluca Campo, Luigi Vignali, Hirosada Yamamoto, Giampaolo Niccoli, Elena Ladich, Giuseppe Biondi-Zoccai, Giulio Guagliumi

43. Three-Year Clinical Outcomes of Everolimus-Eluting Stents From the Post-Marketing Surveillance Study of CobaltChromium Everolimus-Eluting Stent (XIENCE V/PROMUS) in Japan Jiro Aoki, Ken Kozuma, Masaki Awata, Mamoru Nanasato, Nobuo Shiode, Kengo Tanabe, Junichi Yamaguchi, Hajime Kusano, Hong Nie, Takeshi Kimura on behalf of the XIEVCE V/PROMUS PMS Investigators

44. Edge Vascular Response After Resorption of the Everolimus-Eluting Bioresorbable Vascular Scaffold - A 5-Year Serial Optical Coherence Tomography Study -

Hiroki Tateishi, Pannipa Suwannasom, Yohei Sotomi, Shimpei Nakatani, Yuki Ishibashi, Erhan Tenekecioglu, Mohammad Abdelghani, Rafael Cavalcante, Yaping Zeng, Maik J. Grundeken, Felipe N. Albuquerque, Susan Veldhof, Yoshinobu Onuma, Patrick W. Serruys on behalf of the investigators of the ABSORB Cohort B study

45. Impacts of Surgically Performed Renal Denervation on the Cardiovascular and Electrophysiological Variables in the Chronic Atrioventricular Block Dogs - Comparison With Those of Amiodarone Treatment -

Takeshi Wada, Hiroshi Ohara, Yuji Nakamura, Hirofumi Yokoyama, Xin Cao, Hiroko Izumi-Nakaseko, Kentaro Ando, Nobuyuki Murakoshi, Akira Sato, Kazutaka Aonuma, Akira Takahara, Yuji Nakazato, Atsushi Sugiyama

46. Comparison of Chronic Angioscopic Findings of Bare Metal Stents, 1st-Generation Drug-Eluting Stents and 2ndGeneration Drug-Eluting Stents - Multicenter Study of Intra-Coronary Angioscopy After Stent (MICASA) Kazuoki Dai, Hiroshi Matsuoka, Hideo Kawakami, Tetsuya Sato, Kouki Watanabe, Yasuharu Nakama, Masaharu Ishihara

47. Efficacy of 24-Hour Blood Pressure Monitoring in Evaluating Response to Percutaneous Transluminal Renal Angioplasty Kentaro Jujo, Katsumi Saito, Issei Ishida, Yuho Furuki, Taisuke Ouchi, Ahsung Kim, Yuki Suzuki, Haruki Sekiguchi, Junichi Yamaguchi, Hiroshi Ogawa, Nobuhisa Hagiwara

48. Impact of Implantation Technique and Plaque Morphology on Strut Embedment and Scaffold Expansion of Polylactide Bioresorbable Scaffold - Insights From ABSORB Japan Trial Yohei Sotomi, Yoshinobu Onuma, Jouke Dijkstra, Jeroen Eggermont, Shengnan Liu, Erhan Tenekecioglu, Yaping Zeng, Taku Asano, Robbert J. de Winter, Jeffrey J. Popma, Ken Kozuma, Kengo Tanabe, Patrick W. Serruys, Takeshi Kimura 
Cardiac Rehabilitation (1)

49. Regional Clinical Alliance Path and Cardiac Rehabilitation After Hospital Discharge for Acute Myocardial Infarction Patients in Japan - A Nationwide Survey -

Tetsuo Arakawa, Leon Kumasaka, Michio Nakanishi, Masatoshi Nagayama, Hitoshi Adachi, Kozue Ikeda, Kazuteru Fujimoto, Takao Tashiro, Shin-ichi Momomura, Yoichi Goto

Cardiovascular Surgery (19)

50. Preoperative Tissue Doppler Imaging-Derived Atrial Conduction Time Predicts Postoperative Atrial Fibrillation in Patients Undergoing Mitral Valve Surgery for Mitral Valve Regurgitation

Shinya Takahashi, Keijiro Katayama, Masazumi Watanabe, Hiroshi Kodama, Takahiro Taguchi, Tatsuya Kurosaki, Katsuhiko Imai, Taijiro Sueda

51. Impact of Chronic Kidney Disease on Long-Term Outcome of Coronary Artery Bypass Grafting in Patients With Diabetes Mellitus

Suguru Ohira, Kiyoshi Doi, Satoshi Numata, Sachiko Yamazaki, Hidetake Kawajiri, Hitoshi Yaku

52. Characteristics and Risk Factors for Type 2 Endoleak in an East Asian Population From a Japanese Multicenter Database Naoki Fujimura, Hideaki Obara, Kentaro Matsubara, Susumu Watada, Shintaro Shibutani, Takurin Akiyoshi, Hirohisa Harada, Yuko Kitagawa

53. Durability of Mitral Valve Repair Performed Before the Age of 5 Years Makoto Ando, Yukihiro Takahashi

54. Novel Scoring System to Predict Ineligibility for Bridge to Implantable Left Ventricular Assist Device as Destination Therapy Before Extracorporeal Ventricular Assist Device Implantation - For the Coming Era of Destination Therapy in Japan -

Daisuke Nitta, Koichiro Kinugawa, Teruhiko Imamura, Miyoko Endo, Toshiro Inaba, Hisataka Maki, Eisuke Amiya, Masaru Hatano, Osamu Kinoshita, Kan Nawata, Shunei Kyo, Minoru Ono

55. Risk Stratification for Cardiac Allograft Vasculopathy in Heart Transplant Recipients - Annual Intravascular Ultrasound Evaluation -

Takuma Sato, Osamu Seguchi, Hatsue Ishibashi-Ueda, Masanobu Yanase, Norihiro Okada, Kensuke Kuroda, Eriko Hisamatsu, Haruki Sunami, Takuya Watanabe, Seiko Nakajima, Kyoichi Wada, Hiroki Hata, Tomoyuki Fujita, Norihide Fukushima, Junjiro Kobayashi, Takeshi Nakatani

56. Preservation of Mobility of the Posterior Mitral Leaflet After Mitral Valve Repair With Neochordae Using Loop Technique

Hiroto Kitahara, Mitsushige Murata, Kazuma Okamoto, Mikihiko Kudo, Akihiro Yoshitake, Hikaru Tsuruta, Yuji Itabashi, Keiichi Fukuda, Ryohei Yozu, Hideyuki Shimizu

57. Changes in Right Ventricular Volume and Function After Tricuspid Valve Surgery - Tricuspid Annuloplasty vs. Tricuspid Valve Replacement Jae-Woong Choi, Eun-Ah Park, Whal Lee, Kyung-Hwan Kim, Ki-Bong Kim, Hyuk Ahn, Hyung-Kwan Kim, Ho Young Hwang

58. Long-Term Outcomes of Mitral Valve Repair for Active Endocarditis Hideo Kanemitsu, Ken Nakamura, Naoto Fukunaga, Tadaaki Koyama

59. Valve Phenotype and Risk Factors of Aortic Dilatation After Aortic Valve Replacement in Japanese Patients With Bicuspid Aortic Valve Takeshi Kinoshita, Shiho Naito, Tomoaki Suzuki, Tohru Asai

6. Outcome of Tricuspid Valve Plasty in Norwood Stage I Operation Shu-Chien Huang, Yih-Sharng Chen, Chung-I Chang, Ing-Sh Chiu

61. Aortic Valve Pathology in Patients Supported by Continuous-Flow Left Ventricular Assist Device Tomohiro Saito, Katharina Wassilew, Boris Gorodetski, Julia Stein, Volkmar Falk, Thomas Krabatsch, Evgenij Potapov

62. Favorable Effects of Oxygen Inhalation in Patients After Bidirectional Glenn Procedure as Assessed by Cardiovascular Magnetic Resonance Flow Measurement Yu-ichi Ishikawa, Shiro Ishikawa, Ko-ichi Sagawa, Hiroya Ushinohama, Makoto Nakamura, Hideaki Kado

63. Is Bilateral Internal Mammary Arterial Grafting Beneficial for Patients Aged 75 Years or Older? Satoshi Itoh, Naoyuki Kimura, Hideo Adachi, Atsushi Yamaguchi

64. Japanese Multicenter Outcomes With the HeartMate II Left Ventricular Assist Device in Patients With Small Body Surface Area

Minoru Ono, Yoshiki Sawa, Takeshi Nakatani, Ryuji Tominaga, Yoshiro Matsui, Kenji Yamazaki, Yoshikatsu Saiki, Hiroshi Niinami, Goro Matsumiya, Hirokuni Arai on behalf of Japanese HeartMate II Investigators

65. Predictors and Clinical Impacts of Aggravated Left Ventricular Diastolic Dysfunction After Off-Pump Coronary Artery Bypass Grafting

Seung Hyun Lee, Kwan Wook Kim, Hyun-Chel Joo, Kyung-Jong Yoo, Young-Nam Youn

66. Early Results of Simultaneous Transaortic Transcatheter Aortic Valve Implantation and Total Arterial Off-Pump Coronary Artery Revascularization in High-Risk Patients Junjiro Kobayashi, Yusuke Shimahara, Tomoyuki Fujita, Hideaki Kanzaki, Makoto Amaki, Hiroki Hata, Yuta Kume, Kizuku Yamashita, Atsushi Okada 
67. Pre- and Postoperative Predictors of Long-Term Prognosis After Aortic Valve Replacement for Severe Chronic Aortic Regurgitation

Masashi Amano, Chisato Izumi, Sari Imamura, Naoaki Onishi, Jiro Sakamoto, Yodo Tamaki, Soichiro Enomoto, Makoto Miyake, Toshihiro Tamura, Hirokazu Kondo, Kazuaki Kaitani, Kazuo Yamanaka, Yoshihisa Nakagawa

68. Early and Late Outcomes of Surgical Repair for Stanford A Acute Aortic Dissection in Octogenarians

Masato Tochii, Yoshiyuki Takami, Koji Hattori, Hiroshi Ishikawa, Michiko Ishida, Yoshiro Higuchi, Yasushi Takagi

Critical Care (5)

69. Improved Survival With Favorable Neurological Outcome in Elderly Individuals With Out-of-Hospital Cardiac Arrest in Japan - A Nationwide Observational Cohort Study -

Akira Funada, Yoshikazu Goto, Tetsuo Maeda, Ryota Teramoto, Kenshi Hayashi, Masakazu Yamagishi

70. Characteristics and Outcomes of Bath-Related Out-of-Hospital Cardiac Arrest in Japan

Kosuke Kiyohara, Chika Nishiyama, Sumito Hayashida, Tasuku Matsuyama, Toshihiro Hatakeyama, Tomonari Shimamoto, Junichi Izawa, Tomoko Fujii, Yusuke Katayama, Taku Iwami, Tetsuhisa Kitamura

71. Inter-Facility Transfer vs. Direct Admission of Patients With ST-Segment Elevation Acute Myocardial Infarction Undergoing Primary Percutaneous Coronary Intervention

Kenji Nakatsuma, Hiroki Shiomi, Takeshi Morimoto, Yutaka Furukawa, Yoshihisa Nakagawa, Kenji Ando, Kazushige Kadota, Takashi Yamamoto, Satoru Suwa, Minoru Horie, Takeshi Kimura on behalf of the CREDO-Kyoto AMI investigators

72. Impedance Threshold Device Combined With High-Quality Cardiopulmonary Resuscitation Improves Survival With Favorable Neurological Function After Witnessed Out-of-Hospital Cardiac Arrest Atsushi Sugiyama, Sue Duval, Yuji Nakamura, Katsunori Yoshihara, Demetris Yannopoulos

73. Correlation of Pre- and In-Hospital Systolic Blood Pressure in Acute Heart Failure Patients and the Prognostic Implications - Report From the Tokyo Cardiac Care Unit Network Emergency Medical Service Database -

Yasuyuki Shiraishi, Shun Kohsaka, Kazumasa Harada, Takamichi Miyamoto, Shuzou Tanimoto, Kiyoshi Iida, Tetsuro Sakai, Tetsuro Miyazaki, Mayuko Yagawa, Kenichi Matsushita, Shuta Furihata, Naoki Sato, Keiichi Fukuda, Takeshi Yamamoto, Ken Nagao, Morimasa Takayama for the Tokyo CCU Network Scientific Committee

Epidemiology (6)

74. Development of a Risk Equation for the Incidence of Coronary Artery Disease and Ischemic Stroke for Middle-Aged Japanese - Japan Public Health Center-Based Prospective Study -

Hiroshi Yatsuya, Hiroyasu Iso, Yuanying Li, Kazumasa Yamagishi, Yoshihiro Kokubo, Isao Saito, Norie Sawada, Manami Inoue, Shoichiro Tsugane

75. Effects of Aging on the Coagulation Fibrinolytic System in Outpatients of the Cardiovascular Department Akinori Ochi, Taro Adachi, Koichiro Inokuchi, Ko Ogawa, Yuya Nakamura, Yuta Chiba, Shiro Kawasaki, Yoshimi Onishi, Yoshimasa Onuma, Yumi Munetsugu, Hiroyuki Ito, Tatsuya Onuki, Yoshino Minoura, Norikazu Watanabe, Mitsuharu Kawamura, Taku Asano, Youichi Kobayashi

76. The Current Status of Cardiovascular Medicine in Japan - Analysis of a Large Number of Health Records From a Nationwide Claim-Based Database, JROAD-DPC -

Satoshi Yasuda, Kazuhiro Nakao, Kunihiro Nishimura, Yoshihiro Miyamoto, Yoko Sumita, Toshiaki Shishido, Toshihisa Anzai, Hiroyuki Tsutsui, Hiroshi Ito, Issei Komuro, Yoshihiko Saito, Hisao Ogawa on the behalf of JROAD Investigators

77. Calcium-Channel Blockers and Dementia Risk in Older Adults - National Health Insurance Service - Senior Cohort (2002-2013) -

Deri Hwang, Sunyoung Kim, Hangseok Choi, In-Hwan Oh, Byung Sung Kim, Hyun Rim Choi, So Yeon Kim, Chang Won Won

78. Diabetes Trend and Impact on Risk of Cardiovascular Disease in Middle-Aged Japanese People - The CIRCS Study Mina Hayama-Terada, Isao Muraki, Hironori Imano, Masahiko Kiyama, Takeo Okada, Kazumasa Yamagishi, Renzhe Cui, Tetsuya Ohira, Mitsumasa Umesawa, Tomoko Sankai, Shinichi Sato, Akihiko Kitamura, Hiroyasu Iso on behalf of the CIRCS Investigators

79. Depression and Outcomes in Japanese Outpatients With Cardiovascular Disease - A Prospective Observational Study Tsuyoshi Suzuki, Tsuyoshi Shiga, Hisako Omori, Fujio Tatsumi, Katsuji Nishimura, Nobuhisa Hagiwara

Heart Failure (25)

80. Effect of Respiratory Therapy on the Prognosis of Chronic Heart Failure Patients Complicated With Sleep-Disordered Breathing - A Pilot Efficacy Trial -

Hiroyuki Satake, Koichiro Sugimura, Yoshihiro Fukumoto, Koji Fukuda, Makoto Nakano, Masateru Kondo, Shigefumi Fukui, Hiromasa Ogawa, Tsuyoshi Shinozaki, Hiroaki Shimokawa

81. Prognostic Impact of Functional Mitral Regurgitation in Patients Admitted With Acute Decompensated Heart Failure Yuko Wada, Takahiro Ohara, Akira Funada, Takuya Hasegawa, Yasuo Sugano, Hideaki Kanzaki, Hiroyuki Yokoyama, Satoshi Yasuda, Hisao Ogawa, Toshihisa Anzai

82. Influence of Cardiac Function and Loading Conditions on the Myocardial Performance Index - Theoretical Analysis Based on a Mathematical Model Ryo Inuzuka, Seiko Kuwata, Clara Kurishima, Fuyou Liang, Koichi Sughimoto, Hideaki Senzaki 
83. Prognostic Impact of New-Onset Atrial Fibrillation in Patients With Chronic Heart Failure - A Report From the CHART-2 Study -

Takeshi Yamauchi, Yasuhiko Sakata, Masanobu Miura, Soichiro Tadaki, Ryoichi Ushigome, Kenjiro Sato, Takeo Onose, Kanako Tsuji, Ruri Abe, Takuya Oikawa, Shintaro Kasahara, Kotaro Nochioka, Jun Takahashi, Satoshi Miyata, Hiroaki Shimokawa on behalf of the CHART-2 Investigators

84. Determinants and Prognostic Impact of Hyperuricemia in Hospitalization for Acute Heart Failure

Wei-Ming Huang, Pai-Feng Hsu, Hao-Min Cheng, Dai-Yin Lu, Yu-Lun Cheng, Chao-Yu Guo, Shih-Hsien Sung, Wen-Chung Yu, Chen-Huan Chen

85. Low-Normal Serum Sodium and Heart Failure-Related Events in Patients With Heart Failure With Preserved Left Ventricular Ejection Fraction

Hiroaki Kusaka, Seigo Sugiyama, Eiichiro Yamamoto, Eiichi Akiyama, Yasushi Matsuzawa, Yoshihiro Hirata, Koichiro Fujisue, Hirofumi Kurokawa, Junichi Matsubara, Koichi Sugamura, Hirofumi Maeda, Hideaki Jinnouchi, Kunihiko Matsui, Hisao Ogawa

86. Predictors of Worsening Renal Function in Patients With Acute Decompensated Heart Failure Treated by Low-Dose Carperitide

Yuichi Kawase, Kazushige Kadota, Takeshi Tada, Reo Hata, Keiichiro Iwasaki, Takeshi Maruo, Harumi Katoh, Kazuaki Mitsudo

87. Heart Rate Control With If Inhibitor, Ivabradine, in Japanese Patients With Chronic Heart Failure - A Randomized, Double-Blind, Placebo-Controlled Phase II Study -

Hiroyuki Tsutsui, Shinichi Momomura, Akira Yamashina, Hisao Ogawa, Hiroaki Shimokawa, Yasuki Kihara, Yoshihiko Saito, Nobuhisa Hagiwara, Hiroshi Ito, Junya Ako, Takayuki Inomata, Takashi Tanaka, Yasushi Kawasaki on behalf of the study investigators

88. Prognostic Impacts of Metabolic Syndrome in Patients With Chronic Heart Failure - A Multicenter Prospective Cohort Study -

Soichiro Tadaki, Yasuhiko Sakata, Yutaka Miura, Satoshi Miyata, Masanori Asakura, Kazunori Shimada, Takeshi Yamamoto, Yoshihiro Fukumoto, Toshiaki Kadokami, Satoshi Yasuda, Toshiro Miura, Shin-ichi Ando, Masafumi Yano, Masafumi Kitakaze, Hiroyuki Daida, Hiroaki Shimokawa

89. Prognostic Value of Prothrombin Time International Normalized Ratio in Acute Decompensated Heart Failure - A Combined Marker of Hepatic Insufficiency and Hemostatic Abnormality -

Atsushi Okada, Yasuo Sugano, Toshiyuki Nagai, Seiji Takashio, Satoshi Honda, Yasuhide Asaumi, Takeshi Aiba, Teruo Noguchi, Kengo F. Kusano, Hisao Ogawa, Satoshi Yasuda, Toshihisa Anzai on behalf of the NaDEF Investigators

90. Left Ventricular Global Strain for Estimating Relaxation and Filling Pressure - A Multicenter Study -

Taichi Hayashi, Satoshi Yamada, Hiroyuki Iwano, Masahiro Nakabachi, Mamoru Sakakibara, Kazunori Okada, Daisuke Murai, Hisao Nishino, Kenya Kusunose, Kiyotaka Watanabe, Tomoko Ishizu, Kazuaki Wakami, Hirotsugu Yamada, Kaoru Dohi, Yoshihiro Seo, Nobuyuki Ohte, Taisei Mikami, Hiroyuki Tsutsui

Respiratory Collapse of the Inferior Vena Cava Reflects Volume Shift and Subsequent Fluid Refill in Acute Heart Failure Syndrome

Tomohiro Asahi, Marohito Nakata, Namio Higa, Mamoru Manita, Kazuhiko Tabata, Michio Shimabukuro

92. Exhaled Acetone Concentration Is Related to Hemodynamic Severity in Patients With Non-Ischemic Chronic Heart Failure

Tetsuro Yokokawa, Yasuo Sugano, Akito Shimouchi, Atsushi Shibata, Naoya Jinno, Toshiyuki Nagai, Hideaki Kanzaki, Takeshi Aiba, Kengo Kusano, Mikiyasu Shirai, Yasuchika Takeishi, Satoshi Yasuda, Hisao Ogawa, Toshihisa Anzai

93. Value of Virtual Touch Quantification Elastography for Assessing Liver Congestion in Patients With Heart Failure Takashi Yoshitani, Naoya Asakawa, Mamoru Sakakibara, Keiji Noguchi, Yusuke Tokuda, Kiwamu Kamiya, Hiroyuki Iwano, Satoshi Yamada, Yusuke Kudou, Mutsumi Nishida, Chikara Shimizu, Toraji Amano, Hiroyuki Tsutsui

94. Prognostic Impact of Loop Diuretics in Patients With Chronic Heart Failure - Effects of Addition of Renin-AngiotensinAldosterone System Inhibitors and $\beta$-Blockers -

Masanobu Miura, Koichiro Sugimura, Yasuhiko Sakata, Satoshi Miyata, Soichiro Tadaki, Takeshi Yamauchi, Takeo Onose, Kanako Tsuji, Ruri Abe, Takuya Oikawa, Shintaro Kasahara, Kotaro Nochioka, Jun Takahashi, Hiroaki Shimokawa on behalf of the CHART-2 Investigators

95. Prognostic Significance of Insomnia in Heart Failure

Yuki Kanno, Akiomi Yoshihisa, Shunsuke Watanabe, Mai Takiguchi, Tetsuro Yokokawa, Akihiko Sato, Shunsuke Miura, Takeshi Shimizu, Yuichi Nakamura, Satoshi Abe, Takamasa Sato, Satoshi Suzuki, Masayoshi Oikawa, Shu-ichi Saitoh, Yasuchika Takeishi

96. Hippocampal Blood Flow Abnormality Associated With Depressive Symptoms and Cognitive Impairment in Patients With Chronic Heart Failure

Hideaki Suzuki, Yasuharu Matsumoto, Hideki Ota, Koichiro Sugimura, Jun Takahashi, Kenta Ito, Satoshi Miyata, Katsutoshi Furukawa, Hiroyuki Arai, Yoshihiro Fukumoto, Yasuyuki Taki, Hiroaki Shimokawa

97. Relationship of Left Ventricular Diastolic Function to Obesity and Overweight in a Japanese Population With Preserved Left Ventricular Ejection Fraction

Seitetsu L. Lee, Masao Daimon, Marco R. Di Tullio, Shunichi Homma, Tomoko Nakao, Takayuki Kawata, Koichi Kimura, Tomohiro Shinozaki, Megumi Hirokawa, Tomoko S. Kato, Yoshiko Mizuno, Masafumi Watanabe, Yutaka Yatomi, Tsutomu Yamazaki, Issei Komuro 
98. Impaired Mechanics of Left Ventriculo-Atrial Coupling in Patients With Diabetic Nephropathy

Yasuhide Mochizuki, Hidekazu Tanaka, Kensuke Matsumoto, Hiroyuki Sano, Hiroyuki Shimoura, Junichi Ooka,

Takuma Sawa, Keiko Ryo-Koriyama, Yushi Hirota, Wataru Ogawa, Ken-ichi Hirata

99. Transtubular Potassium Concentration Gradient as a Surrogate Measure of Arterial Underfilling in Acute Decompensated Heart Failure

Taiki Sakaguchi, Akio Hirata, Kazunori Kashiwase, Yoshiharu Higuchi, Tomohito Ohtani, Yasushi Sakata, Yukihiro Koretsune, Yoshio Yasumura

100. Diagnostic Value of Right Ventricular Dysfunction in Tachycardia-Induced Cardiomyopathy Using Cardiac Magnetic Resonance Imaging

Atsushi Okada, Ikutaro Nakajima, Yoshiaki Morita, Yuko Y. Inoue, Tsukasa Kamakura, Mitsuru Wada, Kohei Ishibashi, Koji Miyamoto, Hideo Okamura, Satoshi Nagase, Takashi Noda, Takeshi Aiba, Shiro Kamakura, Toshihisa Anzai, Teruo Noguchi, Satoshi Yasuda, Kengo Kusano

101. Sympathetic Nerve Activity Efferent Drive and Beta-Blocker Treatment - Effect of Interaction in Systolic Heart Failure Shuji Joho, Takashi Akabane, Ryuichi Ushijima, Tadakazu Hirai, Koichiro Kinugawa

102. Influence of Left Ventricular Ejection Fraction on the Effects of Supplemental Use of Angiotensin Receptor Blocker Olmesartan in Hypertensive Patients With Heart Failure

Masanobu Miura, Yasuhiko Sakata, Satoshi Miyata, Nobuyuki Shiba, Jun Takahashi, Kotaro Nochioka, Tsuyoshi Takada, Chiharu Saga, Tsuyoshi Shinozaki, Masafumi Sugi, Makoto Nakagawa, Nobuyo Sekiguchi, Tatsuya Komaru, Atsushi Kato, Mitsumasa Fukuchi, Eiji Nozaki, Tetsuya Hiramoto, Kanichi Inoue, Toshikazu Goto, Masatoshi Ohe, Kenji Tamaki, Setsuro Ibayashi, Nobumasa Ishide, Yukio Maruyama, Ichiro Tsuji, Hiroaki Shimokawa on behalf of the SUPPORT Trial Investigators

103. Effect of Overweight and Obesity (Defined by Asian-Specific Cutoff Criteria) on Left Ventricular Diastolic Function and Structure in a General Korean Population

Sung Keun Park, Jae-Hong Ryoo, Chang-Mo Oh, Joong-Myung Choi, Jeong Gyu Kang, Jae-Hon Lee, Ju Youn Chung, Ju Young Jung

104. Vidarabine, an Anti-Herpes Virus Agent, Protects Against the Development of Heart Failure With Relatively Mild SideEffects on Cardiac Function in a Canine Model of Pacing-Induced Dilated Cardiomyopathy

Takashi Nakamura, Takayuki Fujita, Megumi Kishimura, Kenji Suita, Yuko Hidaka, Wenqian Cai, Masanari Umemura, Utako Yokoyama, Masami Uechi, Yoshihiro Ishikawa

Hypertension and Circulatory Control (7)

105. Effect of Diuretic or Calcium-Channel Blocker Plus Angiotensin-Receptor Blocker on Diastolic Function in Hypertensive Patients

Norihisa Toh, Katsuhisa Ishii, Hajime Kihara, Katsuomi Iwakura, Hiroyuki Watanabe, Junichi Yoshikawa, Hiroshi Ito for Effect of ARB/Diuretics on Diastolic Function in Patients with Hypertension 2 (EDEN2) trial investigators

106. Deregulation of Soluble Adhesion Molecules in Resistant Hypertension and Its Role in Cardiovascular Remodeling Ana Paula de Faria, Alessandra Mileni Versuti Ritter, Andréa Rodrigues Sabbatini, Nathália Batista Corrêa, Veridiana Brunelli, Rodrigo Modolo, Heitor Moreno

107. Impact of Renal Denervation on Patients With Obstructive Sleep Apnea and Resistant Hypertension - Insights From the SYMPLICITY HTN-3 Trial -

Kazuomi Kario, Deepak L. Bhatt, David E. Kandzari, Sandeep Brar, John M. Flack, Colleen Gilbert, Suzanne Oparil, Mark Robbins, Raymond R. Townsend, George Bakris

108. Validation of the Korean Genome Epidemiology Study Risk Score to Predict Incident Hypertension in a Large Nationwide Korean Cohort

Nam-Kyoo Lim, Joung-Won Lee, Hyun-Young Park

109. Coexisting Hyponatremia and Decline in Diastolic Blood Pressure Predispose to Atrial Standstill in Hyperkalemic Patients

Takeshi Ideguchi, Toshihiro Tsuruda, Yuji Sato, Kazuo Kitamura

110. Obstructive Sleep Apnea as Possible Causal Factor for Visit-to-Visit Blood Pressure Variability

Kazuki Shiina, Hirofumi Tomiyama, Yoshifumi Takata, Chisa Matsumoto, Mari Odaira, Kota Kato, Tasuku Yamaguchi, Yasuhiro Usui, Akira Yamashina

111. Salt Intake, Home Blood Pressure, and Perinatal Outcome in Pregnant Women

Minako Inoue, Takuya Tsuchihashi, Yasuyuki Hasuo, Masanobu Ogawa, Mitsuhiro Tominaga, Kimika Arakawa, Emi Oishi, Satoko Sakata, Toshio Ohtsubo, Kiyoshi Matsumura, Takanari Kitazono

Imaging (12)

112. Pulmonary Annular Motion Velocity Assessed Using Doppler Tissue Imaging - Novel Echocardiographic Evaluation of Right Ventricular Outflow Tract Function -

Yasunobu Hayabuchi, Akemi Ono, Shoji Kagami

113. Combined Global Longitudinal Strain and Intraventricular Mechanical Dyssynchrony Predicts Long-Term Outcome in Patients With Systolic Heart Failure

Yi-Hsin Chan, Chi-Tai Kuo, Lung-Sheng Wu, Chun-Li Wang, Yung-Hsin Yeh, Lung-An Hsu, Wan-Jing Ho

114. Prognostic Value of Cardiac Sympathetic Nerve Imaging Using Long-Term Follow-up Data - Ischemic vs. Non-Ischemic Heart Failure Etiology -

Shinro Matsuo, Kenichi Nakajima, Tomoaki Nakata 
115. Clinical Feasibility of Simultaneous Acquisition Rest ${ }^{99 \mathrm{~m} T c / S t r e s s}{ }^{201} \mathrm{Tl}$ Dual-Isotope Myocardial Perfusion SinglePhoton Emission Computed Tomography With Semiconductor Camera

Ayano Makita, Naoya Matsumoto, Yasuyuki Suzuki, Yusuke Hori, Keiichiro Kuronuma, Shunichi Yoda, Shu Kasama, Nobuo Iguchi, Yasuhiro Suzuki, Atsushi Hirayama

116. Native T1 Relaxation Time and Extracellular Volume Fraction as Accurate Markers of Diffuse Myocardial Fibrosis in Heart Valve Disease - Comparison With Targeted Left Ventricular Myocardial Biopsy -

Radka Kockova, Petr Kacer, Jan Pirk, Jiri Maly, Lucie Sukupova, Viktor Sikula, Martin Kotrc, Lucia Barciakova, Eva Honsova, Marek Maly, Josef Kautzner, David Sedmera, Martin Penicka

117. Characterization of Compacted Myocardial Abnormalities by Cardiac Magnetic Resonance With Native T1 Mapping in Left Ventricular Non-Compaction Patients - A Comparison With Late Gadolinium Enhancement Hongmei Zhou, Xue Lin, Ligang Fang, Xihai Zhao, Haiyan Ding, Wei Chen, Ruiyi Xu, Xiaoyin Bai, Yining Wang, Quan Fang

118. Diagnostic Performance of a Cadmium-Zinc-Telluride Single-Photon Emission Computed Tomography System With Low-Dose Technetium-99 m as Assessed by Fractional Flow Reserve Taishiro Chikamori, Satoshi Hida, Nobuhiro Tanaka, Yuko Igarashi, Jun Yamashita, Chie Shiba, Naotaka Murata, Kou Hoshino, Yohei Hokama, Akira Yamashina

119. Relationship Between Thickness of Calcium on Optical Coherence Tomography and Crack Formation After Balloon Dilatation in Calcified Plaque Requiring Rotational Atherectomy Nobuhiko Maejima, Kiyoshi Hibi, Kenichiro Saka, Eiichi Akiyama, Masaaki Konishi, Mitsuaki Endo, Noriaki Iwahashi, Kengo Tsukahara, Masami Kosuge, Toshiaki Ebina, Satoshi Umemura, Kazuo Kimura

Micro-Computed Tomography for the Quantitative 3-Dimensional Assessment of the Compact Myocardium in the Mouse Embryo

Samer S. Merchant, Yasuhiro Kosaka, H. Joseph Yost, Edward W. Hsu, Luca Brunelli

121. Optical Coherence Tomography of De Novo Lesions and In-Stent Restenosis in Coronary Saphenous Vein Grafts (OCTOPUS Study)

Tomasz Roleder, Elżbieta Pociask, Wojciech Wańha, Magdalena Dobrolińska, Paweł Gąsior, Grzegorz Smolka, Wojciech Walkowicz, Tomasz Jadczyk, Tomasz Bochenek, Dariusz Dudek, Andrzej Ochała, Katarzyna Mizia-Stec, Zbigniew Gąsior, Michał Tendera, Ziad A Ali, Wojciech Wojakowski

122. Optimal Scan Time for Single-Phase Myocardial Computed Tomography Perfusion to Detect Myocardial Ischemia - Derivation Cohort From Dynamic Myocardial Computed Tomography Perfusion -

Yuki Tanabe, Teruhito Kido, Akira Kurata, Teruyoshi Uetani, Naoki Fukuyama, Takahiro Yokoi, Hikaru Nishiyama, Tomoyuki Kido, Masao Miyagawa, Teruhito Mochizuki

123. Magnetic Resonance Imaging of Reverse Remodeling After Coronary Revascularization in Ischemic Heart Disease - Morphologic Evaluation -

Eun-Ah Park, Whal Lee, Se-Jin Oh, Ki-Bong Kim

Ischemic Heart Disease (33)

124. Glucose Fluctuations Aggravate Cardiac Susceptibility to Ischemia/Reperfusion Injury by Modulating MicroRNAs Expression

Shotaro Saito, Luong Cong Thuc, Yasushi Teshima, Chisato Nakada, Satoru Nishio, Hidekazu Kondo, Akira Fukui, Ichitaro Abe, Yuki Ebata, Tetsunori Saikawa, Masatsugu Moriyama, Naohiko Takahashi

125. Epicardial Adipose Tissue Accumulation Is Associated With Renal Dysfunction and Coronary Plaque Morphology on Multidetector Computed Tomography

Koki Nakanishi, Shota Fukuda, Atsushi Tanaka, Kenichiro Otsuka, Haruyuki Taguchi, Junichi Yoshikawa, Kenei Shimada

126. Glycemic Variability on Continuous Glucose Monitoring System Correlates With Non-Culprit Vessel Coronary Plaque Vulnerability in Patients With First-Episode Acute Coronary Syndrome - Optical Coherence Tomography Study -

Masaomi Gohbara, Kiyoshi Hibi, Takayuki Mitsuhashi, Nobuhiko Maejima, Noriaki Iwahashi, Shunsuke Kataoka, Eiichi Akiyama, Kengo Tsukahara, Masami Kosuge, Toshiaki Ebina, Satoshi Umemura, Kazuo Kimura

127. Optimal Medical Therapy vs. Percutaneous Coronary Intervention for Patients With Coronary Chronic Total Occlusion - A Propensity-Matched Analysis -

Jeong Hoon Yang, Bum Sung Kim, Woo Jin Jang, Joonghyun Ahn, Taek Kyu Park, Young Bin Song, Joo-Yong Hahn, Jin-Ho Choi, Sang Hoon Lee, Hyeon-Cheol Gwon, Seung-Hyuk Choi

128. Factors Affecting Platelet Reactivity 2 Hours After P2 $\mathrm{Y}_{12}$ Receptor Antagonist Loading in Primary Percutaneous Coronary Intervention for ST-Elevation Myocardial Infarction - Impact of Pain-to-Loading Time Ioanna Xanthopoulou, Periklis Davlouros, Grigorios Tsigkas, Nikolaos Koutsogiannis, Sotirios Patsilinakos, Spyridon Deftereos, George Hahalis, Dimitrios Alexopoulos

129. Effects of the Addition of Eicosapentaenoic Acid to Strong Statin Therapy on Inflammatory Cytokines and Coronary Plaque Components Assessed by Integrated Backscatter Intravascular Ultrasound Toshiyuki Niki, Tetsuzo Wakatsuki, Koji Yamaguchi, Yoshio Taketani, Hiroyasu Oeduka, Kenya Kusunose, Takayuki Ise, Takashi Iwase, Hirotsugu Yamada, Takeshi Soeki, Masataka Sata

130. Effect of Statin Treatment and Low-Density Lipoprotein-Cholesterol on Short-Term Mortality in Acute Myocardial Infarction Patients Undergoing Primary Percutaneous Coronary Intervention - Multicenter Registry From Tokyo CCU Network Database -

Mizuki Miura, Masao Yamasaki, Yukari Uemura, Masatomo Yoshikawa, Katsumi Miyauchi, Hiroyuki Tanaka, Hideki Miyachi, Jun Yamashita, Makoto Suzuki, Takeshi Yamamoto, Ken Nagao, Issei Komuro, Morimasa Takayama 
131. Plasma Glucagon-Like Peptide-1 and Tissue Characteristics of Coronary Plaque in Non-Diabetic Acute Coronary Syndrome Patients

Takayuki Mitsuhashi, Kiyoshi Hibi, Masaaki Konishi, Nobuhiko Maejima, Noriaki Iwahashi, Kengo Tsukahara, Masami Kosuge, Toshiaki Ebina, Satoshi Umemura, Kazuo Kimura

132. Intravascular Ultrasound Guidance vs. Angiographic Guidance in Primary Percutaneous Coronary Intervention for STSegment Elevation Myocardial Infarction - Long-Term Clinical Outcomes From the CREDO-Kyoto AMI Registry -

Kenji Nakatsuma, Hiroki Shiomi, Takeshi Morimoto, Kenji Ando, Kazushige Kadota, Hiroki Watanabe, Tomohiko Taniguchi, Takashi Yamamoto, Yutaka Furukawa, Yoshihisa Nakagawa, Minoru Horie, Takeshi Kimura on behalf of the CREDO-Kyoto AMI investigators

133. Long-Term Survival Benefit of Coronary Revascularization in Patients Undergoing Stress Myocardial Perfusion Imaging Mario Petretta, Wanda Acampa, Stefania Daniele, Emilia Zampella, Roberta Assante, Carmela Nappi, Marco Salvatore, Alberto Cuocolo

134. Additive Value of Heart Rate Variability in Predicting Obstructive Coronary Artery Disease Beyond Framingham Risk
Hsin-Ru Li, Tse-Min Lu, Hao-Min Cheng, Dai-Yin Lu, Chuen-Wang Chiou, Shao-Yuan Chuang, Albert C. Yang, Shih-Hsien Sung, Wen-Chung Yu, Chen-Huan Chen

135. Shock Index as a Predictor of Myocardial Damage and Clinical Outcome in ST-Elevation Myocardial Infarction
Sebastian J Reinstadler, Georg Fuernau, Charlotte Eitel, Suzanne de Waha, Steffen Desch, Bernhard Metzler, Gerhard

135. Shock Index as a Predictor of Myocardial Damage and Clinical Outcome in ST-Elevation Myocardial Infarction
Sebastian J Reinstadler, Georg Fuernau, Charlotte Eitel, Suzanne de Waha, Steffen Desch, Bernhard Metzler, Gerhard
Schuler

135. Shock Index as a Predictor of Myocardial Damage and Clinical Outcome in ST-Elevation Myocardial Infarction
Sebastian J Reinstadler, Georg Fuernau, Charlotte Eitel, Suzanne de Waha, Steffen Desch, Bernhard Metzler, Gerhard
Schuler, Holger Thiele, Ingo Eitel

136. Elevated Levels of Serum Fibrin and Fibrinogen Degradation Products Are Independent Predictors of Larger Coronary Plaques and Greater Plaque Necrotic Core Michel T Corban, Olivia Y Hung, Girum Mekonnen, Parham Eshtehardi, Danny J Eapen, Emad Rasoul-Arzrumly, Hatem Al Kassem, Pankaj Manocha, Yi-An Ko, Laurence S Sperling, Arshed A Quyyumi, Habib Samady

137. Radiation-Dose-Lowering Effects of Landiolol Hydrochloride in Coronary Angiography Using Computed Tomography (DELIGHT) - A Prospective Multicenter Study -

Teruhito Kido, Teruhito Mochizuki, Masaharu Hirano, Yoshitake Yamada, Ryoichi Tanaka, Suzu Kanzaki, Masahiro Higashi, Masahiro Jinzaki, Kunihiro Yoshioka, Sachio Kuribayashi

138. Impact of Low Diastolic Blood Pressure on Risk of Cardiovascular Death in Elderly Patients With Coronary Artery Disease After Revascularization - The CREDO-Kyoto Registry Cohort-1 -

Hisashi Kai, Takeshi Kimura, Kenji Fukuda, Yoshihiro Fukumoto, Tatsuyuki Kakuma, Yutaka Furukawa on behalf of CREDO-Kyoto Investigators

139. Clinical Impact of the Cardio-Ankle Vascular Index for Predicting Cardiovascular Events After Acute Coronary Syndrome

Masaomi Gohbara, Noriaki Iwahashi, Yuka Sano, Eiichi Akiyama, Nobuhiko Maejima, Kengo Tsukahara, Kiyoshi Hibi, Masami Kosuge, Toshiaki Ebina, Satoshi Umemura, Kazuo Kimura

140. Multicenter Cohort Study of Acute Myocardial Infarction in Korea - Interim Analysis of the Korea Acute Myocardial Infarction Registry-National Institutes of Health Registry -

Ju Han Kim, Shung-Chull Chae, Dong Joo Oh, Hyo-Soo Kim, Young Jo Kim, Youngkeun Ahn, Myeong Chan Cho, Chong Jin Kim, Jung-Han Yoon, Hyun-Young Park, Myung Ho Jeong, Korea Acute Myocardial Infarction-National Institutes of Health Registry Investigators

141. Initial Total Bilirubin and Clinical Outcome in Patients With ST-Segment Elevation Myocardial Infarction Undergoing Primary Percutaneous Coronary Intervention With Drug-Eluting Stents

Sang-Ryul Chung, Tae-Hyun Yang, Ho-Cheol Shin, Han-Young Jin, Jeong-Sook Seo, Jae-Sik Jang, Dae-Kyeong Kim, Dong-Soo Kim, Gwang-Won Seo, Pil-Sang Song, Dong-Kie Kim, Ki-Hun Kim, Sang-Hoon Seol, Doo-Il Kim, Yun-Kyeong Cho, Hyuk-Joon Yoon, Chang-Wook Nam, Seung-Ho Hur, Ung Kim, Jong-Seon Park, Young-Jo Kim

142. Impact of Age on the Functional Significance of Intermediate Epicardial Artery Disease

Xiongjie Jin, Hong-Seok Lim, Seung-Jea Tahk, Hyoung-Mo Yang, Myeong-Ho Yoon, So-Yeon Choi, Byoung-Joo Choi, Andy S. C. Yong, William F. Fearon, Seung Soo Sheen, Kyoung-Woo Seo, Joon-Han Shin

143. Effect of Smoking Status on Clinical Outcome and Efficacy of Clopidogrel in Acute Coronary Syndrome Masaki Kodaira, Hiroaki Miyata, Yohei Numasawa, Ikuko Ueda, Yuichiro Maekawa, Koichiro Sueyoshi, Shiro Ishikawa, Takahiro Ohki, Kouji Negishi, Keiichi Fukuda, Shun Kohsaka

144. Clinical Spectrum and Outcome of Patients With Non-ST-Segment Elevation Acute Coronary Syndrome and No Obstructive Coronary Atherosclerosis

Gaetano A. Lanza, Giulia Careri, Alessandra Stazi, Angelo Villano, Antonio De Vita, Cristina Aurigemma, Filippo Crea

145. Higher Hemoglobin A1c After Discharge Is an Independent Predictor of Adverse Outcomes in Patients With Acute Coronary Syndrome - Findings From the PACIFIC Registry -

Keiji Noguchi, Mamoru Sakakibara, Naoya Asakawa, Yusuke Tokuda, Kiwamu Kamiya, Takashi Yoshitani, Koji Oba, Katsumi Miyauchi, Yuji Nishizaki, Hisao Ogawa, Hiroyoshi Yokoi, Masayasu Matsumoto, Masafumi Kitakaze, Takeshi Kimura, Tetsuo Matsubara, Yuji Ikari, Kazuo Kimura, Hideki Origasa, Takaaki Isshiki, Yoshihiro Morino, Hiroyuki Daida, Hiroyuki Tsutsui on behalf of the PACIFIC investigators

146. Transient New-Onset Atrial Fibrillation Is Associated With Poor Clinical Outcomes in Patients With Acute Myocardial Infarction Jin Wi, Dong-Ho Shin, Jung-Sun Kim, Byeong-Keuk Kim, Young-Guk Ko, Donghoon Choi, Myeong-Ki Hong, Yangsoo Jang 
147. Time to Reperfusion in ST-Segment Elevation Myocardial Infarction Patients With vs. Without Pre-Hospital Mobile Telemedicine 12-Lead Electrocardiogram Transmission

Shoji Kawakami, Yoshio Tahara, Teruo Noguchi, Nobuhito Yagi, Yu Kataoka, Yasuhide Asaumi, Michio Nakanishi, Yoichi Goto, Hiroyuki Yokoyama, Hiroshi Nonogi, Hisao Ogawa, Satoshi Yasuda

148. Effects of 4 Statins on Regression of Coronary Plaque in Acute Coronary Syndrome

Kensuke Matsushita, Kiyoshi Hibi, Naohiro Komura, Eiichi Akiyama, Nobuhiko Maejima, Noriaki Iwahashi, Kengo

Tsukahara, Masami Kosuge, Toshiaki Ebina, Shinichi Sumita, Satoshi Umemura, Kazuo Kimura

149. Impact of Pressure Signal Drift on Fractional Flow Reserve-Based Decision-Making for Patients With Intermediate Coronary Artery Stenosis

Nobutaka Wakasa, Tatsuhiko Kuramochi, Naoto Mihashi, Noriko Terada, Yoshihisa Kanaji, Tadashi Murai, Tetsumin Lee, Taishi Yonetsu, Kazuhiko Kobashi, Kazunori Miyamoto, Hiroshi Tobata, Tsunekazu Kakuta

150. Intracoronary Acetylcholine Provocation Testing - Omission of the 20- $\mu \mathrm{g}$ Dose Is Feasible in Patients Without Coronary Artery Spasm in the Other Coronary Artery -

Yuichi Saito, Hideki Kitahara, Toshihiro Shoji, Satoshi Tokimasa, Takashi Nakayama, Kazumasa Sugimoto, Yoshihide Fujimoto, Yoshio Kobayashi

151. Deletion of CD28 Co-stimulatory Signals Exacerbates Left Ventricular Remodeling and Increases Cardiac Rupture After Myocardial Infarction

Akihiko Kubota, Hiroshi Hasegawa, Hiroyuki Tadokoro, Masanori Hirose, Yuka Kobara, Tomoko Yamada-Inagawa, Genzou Takemura, Yoshio Kobayashi, Hiroyuki Takano

152. Impact of Malondialdehyde-Modified Low-Density Lipoprotein on Tissue Characteristics in Patients With Stable Coronary Artery Disease - Integrated Backscatter-Intravascular Ultrasound Study -

Hiroki Ikenaga, Satoshi Kurisu, Shingo Kono, Yoji Sumimoto, Noriaki Watanabe, Takashi Shimonaga, Tadanao Higaki, Toshitaka Iwasaki, Naoya Mitsuba, Ken Ishibashi, Yoshihiro Dohi, Yukihiro Fukuda, Yasuki Kihara

153. Circulating miR-122-5p/miR-133b Ratio Is a Specific Early Prognostic Biomarker in Acute Myocardial Infarction Nuno Cortez-Dias, Marina C. Costa, Pedro Carrilho-Ferreira, Doroteia Silva, Cláudia Jorge, Carina Calisto, Teresa Pessoa, Susana Robalo Martins, João Carvalho de Sousa, Pedro Canas da Silva, Manuela Fiúza, António Nunes Diogo, Fausto J. Pinto, Francisco J. Enguita

154. Prevalence and Distribution of Coronary Artery Calcification in Asymptomatic United States and Korean Adults - CrossSectional Propensity-Matched Analysis -

Donghee Han, Bríain ó Hartaigh, Heidi Gransar, Ji Hyun Lee, Su-Yeon Choi, Eun Ju Chun, Jidong Sung, Hae-Won Han, Sung Hak Park, Tracy Callister, Fay Y. Lin, James K. Min, Hyuk-Jae Chang

155. Warranty Period of Zero Coronary Artery Calcium Score for Predicting All-Cause Mortality According to Cardiac Risk Burden in Asymptomatic Korean Adults Ji Hyun Lee, Donghee Han, Bríain ó Hartaigh, Asim Rizvi, Heidi Gransar, Hyung-Bok Park, Hyo Eun Park, Su-Yeon Choi, Eun Ju Chun, Jidong Sung, Sung Hak Park, Hae-Won Han, James K. Min, Hyuk-Jae Chang

156. Pharmacodynamic Assessment of Platelet Reactivity After a Loading Dose of Prasugrel or Clopidogrel in Patients With ST-Segment Elevation Myocardial Infarction

Shinya Ichikawa, Kengo Tsukahara, Yugo Minamimoto, Yuichiro Kimura, Yasushi Matsuzawa, Nobuhiko Maejima, Noriaki Iwahashi, Kiyoshi Hibi, Masami Kosuge, Toshiaki Ebina, Kazuo Kimura

Metabolic Disorder (3)

157. Pentraxin 3 and Platelet Activation in Obese Patients After Gastric Banding Francesca Santilli, Maria Teresa Guagnano, Paolo Innocenti, Liberato Aceto, Natale Vazzana, Stefano Lattanzio, Rossella Liani, Romina Tripaldi, Valeria Creato, Mario Romano, Giovanni Davì

158. Lipoprotein(a) in Familial Hypercholesterolemia With Proprotein Convertase Subtilisin/Kexin Type 9 (PCSK9) Gain-ofFunction Mutations

Hayato Tada, Masa-aki Kawashiri, Taiji Yoshida, Ryota Teramoto, Atsushi Nohara, Tetsuo Konno, Akihiro Inazu, Hiroshi Mabuchi, Masakazu Yamagishi, Kenshi Hayashi

159. Efficacy and Safety of Alirocumab in Japanese Patients With Heterozygous Familial Hypercholesterolemia or at High Cardiovascular Risk With Hypercholesterolemia Not Adequately Controlled With Statins - ODYSSEY JAPAN Randomized Controlled Trial -

Tamio Teramoto, Masahiko Kobayashi, Hiromi Tasaki, Hiroaki Yagyu, Toshinori Higashikata, Yoshiharu Takagi, Kiyoko Uno, Marie T. Baccara-Dinet, Atsushi Nohara

Molecular Cardiology (2)

160. Next Generation Sequencing and Linkage Analysis for the Molecular Diagnosis of a Novel Overlapping Syndrome Characterized by Hypertrophic Cardiomyopathy and Typical Electrical Instability of Brugada Syndrome

Ruggiero Mango, Andrea Luchetti, Raffaele Sangiuolo, Valentina Ferradini, Nicola Briglia, Emiliano Giardina, Fabrizio Ferrè, Manuela Helmer Citterich, Francesco Romeo, Giuseppe Novelli, Federica Sangiuolo

161. Suppressive Effects of Glucose-Dependent Insulinotropic Polypeptide on Cardiac Hypertrophy and Fibrosis in Angiotensin II-Infused Mouse Models Munenori Hiromura, Yusaku Mori, Kyoko Kohashi, Michishige Terasaki, Kyoko Shinmura, Takaharu Negoro, Hikaru Kawashima, Mao Kogure, Toshimi Wachi, Rena Watanabe, Kengo Sato, Hideki Kushima, Masako Tomoyasu, Yasuko Nakano, Yuichiro Yamada, Takuya Watanabe, Tsutomu Hirano 
Myocardial Disease (8)

162. Elevated Cardiac Enzymes in Hypertrophic Cardiomyopathy Patients With Heart Failure - A 20-Year Prospective Follow-up Study -

Mareomi Hamada, Yuji Shigematsu, Takashi Ohtani, Shuntaro Ikeda

163. Extent of Late Gadolinium Enhancement on Cardiac Magnetic Resonance Imaging in Japanese Hypertrophic Cardiomyopathy Patients

Yasuki Hen, Nobuo Iguchi, Yuko Utanohara, Kaori Takada, Haruhiko Machida, Ayako Takara, Kunihiko Teraoka, Tetsuya Sumiyoshi, Itaru Takamisawa, Morimasa Takayama, Tsutomu Yoshikawa

164. Prevalence and Clinical Features of Focal Takotsubo Cardiomyopathy

Ken Kato, Hideki Kitahara, Yoshihide Fujimoto, Yoshiaki Sakai, Iwao Ishibashi, Toshiharu Himi, Yoshio Kobayashi

165. Left Atrial Morphology, Size and Function in Patients With Transthyretin Cardiac Amyloidosis and Primary Hypertrophic Cardiomyopathy - Comparative Strain Imaging Study -

Cesare de Gregorio, Giuseppe Dattilo, Matteo Casale, Anna Terrizzi, Rocco Donato, Gianluca Di Bella

166. Quantitative Comparison Between Amyloid Deposition Detected by ${ }^{99 m T c-D i p h o s p h o n a t e ~ I m a g i n g ~ a n d ~ M y o c a r d i a l ~}$ Deformation Evaluated by Strain Echocardiography in Transthyretin-Related Cardiac Amyloidosis

Gianluca Di Bella, Fabio Minutoli, Paolo Piaggi, Matteo Casale, Anna Mazzeo, Concetta Zito, Giuseppe Oreto, Sergio Baldari, Giuseppe Vita, Alessandro Pingitore, Bijoy K. Khandheria, Scipione Carerj

167. Impact of Malignancies in the Early and Late Time Course of Takotsubo Cardiomyopathy

Mélanie Girardey, Laurence Jesel, Umberto Campia, Nathan Messas, Sébastien Hess, Alessio Imperiale, Cyrille Blondet, Annie Trinh, Patrick Ohlmann, Olivier Morel

168. Single-Coil Defibrillator Leads Yield Satisfactory Defibrillation Safety Margin in Hypertrophic Cardiomyopathy Hideo Okamura, Paul A. Friedman, Yuko Inoue, Takashi Noda, Takeshi Aiba, Satoshi Yasuda, Hisao Ogawa, Shiro Kamakura, Kengo Kusano, Raul E. Espinosa

169. Heart Failure Progression in Hypertrophic Cardiomyopathy - Possible Insights From Cardiopulmonary Exercise Testing Damiano Magrì, Federica Re, Giuseppe Limongelli, Piergiuseppe Agostoni, Elisabetta Zachara, Michele Correale, Vittoria Mastromarino, Caterina Santolamazza, Matteo Casenghi, Giuseppe Pacileo, Fabio Valente, Marco Morosin, Beatrice Musumeci, Erika Pagannone, Antonello Maruotti, Massimo Uguccioni, Massimo Volpe, Camillo Autore

Pediatric Cardiology and Adult Congenital Heart Disease (13)

170. Treat and Repair Strategy in Patients With Atrial Septal Defect and Significant Pulmonary Arterial Hypertension Yasufumi Kijima, Teiji Akagi, Yoichi Takaya, Satoshi Akagi, Koji Nakagawa, Kengo Kusano, Shunji Sano, Hiroshi Ito

171. Pediatric Cohort With Long QT Syndrome - KCNH2 Mutation Carriers Present Late Onset But Severe Symptoms Junichi Ozawa, Seiko Ohno, Takashi Hisamatsu, Hideki Itoh, Takeru Makiyama, Hiroshi Suzuki, Akihiko Saitoh, Minoru Horie

172. Lipopolysaccharide Delays Closure of the Rat Ductus Arteriosus by Induction of Inducible Nitric Oxide Synthase But Not Prostaglandin E2

Ichige Kajimura, Toru Akaike, Susumu Minamisawa

173. Vascular Function and Myocardial Performance Indices in Children Born Small for Gestational Age Maria Felicia Faienza, Giacomina Brunetti, Maurizio Delvecchio, Annapaola Zito, Fabrizia De Palma, Francesca Cortese, Adriana Nitti, Elena Massari, Michele Gesualdo, Gabriella Ricci, Santa Carbonara, Paola Giordano, Luciano Cavallo, Pietro Scicchitano, Marco Matteo Ciccone

174. Nationwide Survey of the Transfer of Adults With Congenital Heart Disease From Pediatric Cardiology Departments to Adult Congenital Heart Disease Centers in Japan

Ryota Ochiai, Hitoshi Kato, Naomi Akiyama, Fukiko Ichida, Atsushi Yao, Ryo Inuzuka, Koichiro Niwa, Isao Shiraishi, Toshio Nakanishi

175. High Incidence of Dilated Cardiomyopathy After Right Ventricular Inlet Pacing in Patients With Congenital Complete Atrioventricular Block

Nobuyuki Tsujii, Aya Miyazaki, Heima Sakaguchi, Koji Kagisaki, Tetsuya Yamamoto, Michio Matsuoka, Yuriko Shima, Hajime Ichikawa, Hideo Ohuchi

176. Trunk-to-Peripheral Fat Ratio Predicts Subsequent Blood Pressure Levels in Pubertal Children With Relatively Low Body Fat - Three-Year Follow-up Study -

Katsuyasu Kouda, Kumiko Ohara, Yuki Fujita, Harunobu Nakamura, Masayuki Iki

177. Frequency of Miscarriage/Stillbirth and Terminations of Pregnancy Among Women With Congenital Heart Disease in Germany, Hungary and Japan

Marc-André Koerten, Koichiro Niwa, András Szatmári, Balint Hajnalka, Zoltán Ruzsa, Nicole Nagdyman, Eva Niggemeyer, Brigitte Peters, Karl-Theodor M. Schneider, Bettina Kuschel, Yoshiko Mizuno, Felix Berger, Harald Kaemmerer, Ulrike M. M. Bauer

178. Cardiovascular Remodeling and Dysfunction Across a Range of Growth Restriction Severity in Small for Gestational Age Infants - Implications for Fetal Programming -

Yohei Akazawa, Akira Hachiya, Shoko Yamazaki, Yoichiro Kawasaki, Chizuko Nakamura, Yusuke Takeuchi, Mai Kusakari, Yukihide Miyosawa, Motoko Kamiya, Noriko Motoki, Kenichi Koike, Tomohiko Nakamura

179. Beta-Blockers and Fetal Growth Restriction in Pregnant Women With Cardiovascular Disease Kayo Tanaka, Hiroaki Tanaka, Chizuko Kamiya, Shinji Katsuragi, Masami Sawada, Mitsuhiro Tsuritani, Masashi Yoshida, Naoko Iwanaga, Jun Yoshimatsu, Tomoaki Ikeda 
180. Clinical Features of Acute and Fulminant Myocarditis in Children - 2nd Nationwide Survey by Japanese Society of Pediatric Cardiology and Cardiac Surgery -

Hiroyuki Matsuura, Fukiko Ichida, Tsutomu Saji, Shunichi Ogawa, Kenji Waki, Masahide Kaneko, Masahiro Tahara, Takashi Soga, Yasuo Ono, Satoshi Yasukochi

181. Decreased Aortic Elasticity in Children With Marfan Syndrome or Loeys-Dietz Syndrome

Yohei Akazawa, Noriko Motoki, Akira Tada, Shoko Yamazaki, Akira Hachiya, Satoshi Matsuzaki, Motoko Kamiya, Tomohiko Nakamura, Tomoki Kosho, Yuji Inaba

182. Serum Tenascin-C as a Novel Predictor for Risk of Coronary Artery Lesion and Resistance to Intravenous Immunoglobulin in Kawasaki Disease - A Multicenter Retrospective Study -

Yoshiaki Okuma, Kenji Suda, Hideyuki Nakaoka, Yasuhiro Katsube, Yoshihide Mitani, Yukako Yoshikane, Fukiko Ichida, Takeji Matsushita, Hiroyuki Shichino, Isao Shiraishi, Jun Abe, Michiaki Hiroe, Toshimichi Yoshida, Kyoko Imanaka-Yoshida

Peripheral Vascular Disease (8)

183. Paramalleolar Arterial Bollinger Score in the Era of Diabetes and End-Stage Renal Disease - Usefulness for Predicting Operative Outcome of Critical Limb Ischemia -

Mitsuru Matsukura, Katsuyuki Hoshina, Kunihiro Shigematsu, Tetsuro Miyata, Toshiaki Watanabe

184. Baseline Characterization of Japanese Peripheral Arterial Disease Patients - Analysis of Surveillance of Cardiovascular Events in Antiplatelet-Treated Arteriosclerosis Obliterans Patients in Japan (SEASON) -

Yukihito Higashi, Tetsuro Miyata, Hiroshi Shigematsu, Hideki Origasa, Masatoshi Fujita, Hiroshi Matsuo, Hiroaki Naritomi, Hiroaki Matsuda, Masahide Nakajima for the SEASON Investigators

185. Clinical Outcome of Surgical Endarterectomy for Common Femoral Artery Occlusive Disease Sosei Kuma, Kiyoshi Tanaka, Takahiro Ohmine, Koichi Morisaki, Akio Kodama, Atsushi Guntani, Masaru Ishida, Jin Okazaki, Shinsuke Mii

186. Impact of Noninvasive Conservative Medical Treatment for Symptomatic Isolated Celiac Artery Dissection Toshihisa Ichiba, Masahiko Hara, Keiji Yunoki, Masaki Urashima, Hiroshi Naitou

187. Evolution of Computed Tomographic Characteristics of Spontaneous Isolated Superior Mesenteric Artery Dissection During Conservative Management

Koichi Tomita, Hideaki Obara, Yasuhito Sekimoto, Kentaro Matsubara, Susumu Watada, Naoki Fujimura, Shintaro Shibutani, Kazuhito Nagasaki, Shinobu Hayashi, Hirohisa Harada, Atsunori Asami, Norio Uchida, Toshihiro Kakefuda, Yuko Kitagawa

188. Raison d'etre of Tibial Artery Bypass for Intermittent Claudication in the Era of Endovascular Therapy

Shinsuke Mii, Kiyoshi Tanaka, Ryoichi Kyuragi, Sosei Kuma, Akio Kodama, Ryota Fukunaga, Ichiro Masaki, Jin Okazaki, Daihiko Eguchi, Terutoshi Yamaoka, Akira Mori, Atsushi Guntani, Jun Okadome

189. Change in Ankle-Brachial Index Over Time in a Screened Japanese Cohort - The Okinawa Peripheral Arterial Disease Study -

Yuichiro Toma, Akio Ishida, Kozen Kinjo, Yusuke Ohya

190. Outcomes of Critical Limb Ischemia in Hemodialysis Patients After Distal Bypass Surgery - Poor Limb Prognosis With Stage 4 Wound, Ischemia, and Foot Infection (WIfI) -

Katsuyuki Hoshina, Kota Yamamoto, Tetsuro Miyata, Toshiaki Watanabe

Preventive Medicine (1)

191. Results of a Prospective Study of Acute Coronary Syndrome Hospitalization After Enactment of a Smoking Ban in Public Places in Hyogo Prefecture - Comparison With Gifu, a Prefecture Without a Public Smoking Ban -

Yukihito Sato, Shinya Minatoguchi, Kazuhiko Nishigaki, Ken-ichi Hirata, Tohru Masuyama, Yutaka Furukawa, Masaaki Uematsu, Junichi Yoshikawa, Satoru Otsuji, Mami Iida, Hisayoshi Fujiwara for the SHYOGI Study Investigators

Pulmonary Circulation (11)

192. Prognostic Factors for Survival in Pulmonary Hypertension Due to Left Heart Disease Sayuri Yamabe, Yoshihiro Dohi, Shinya Fujisaki, Akifumi Higashi, Hiroki Kinoshita, Yoshiharu Sada, Takayuki Hidaka, Satoshi Kurisu, Hideya Yamamoto, Yasuki Kihara

193. Coagulation-Fibrinolysis System and Postoperative Outcomes of Patients With Chronic Thromboembolic Pulmonary Hypertension

Fumiaki Kato, Nobuhiro Tanabe, Keiichi Ishida, Rika Suda, Ayumi Sekine, Rintaro Nishimura, Takayuki Jujo, Toshihiko Sugiura, Seiichiro Sakao, Koichiro Tatsumi

194. Multiple Beneficial Effects of Balloon Pulmonary Angioplasty in Patients With Chronic Thromboembolic Pulmonary Hypertension

Shunsuke Tatebe, Koichiro Sugimura, Tatsuo Aoki, Masanobu Miura, Kotaro Nochioka, Nobuhiro Yaoita, Hideaki Suzuki, Haruka Sato, Saori Yamamoto, Kimio Satoh, Yoshihiro Fukumoto, Hiroaki Shimokawa

195. Detrimental Impact of Vasopressin V2 Receptor Antagonism in a SU5416/Hypoxia/Normoxia-Exposed Rat Model of Pulmonary Arterial Hypertension Itaru Goto, Kaoru Dohi, Yoshito Ogihara, Ryuji Okamoto, Norikazu Yamada, Yoshihide Mitani, Masaaki Ito

196. Mean Pulmonary Artery Pressure Using Echocardiography in Chronic Thromboembolic Pulmonary Hypertension Hajime Kasai, Akane Matsumura, Toshihiko Sugiura, Ayako Shigeta, Nobuhiro Tanabe, Keiko Yamamoto, Hideki Miwa, Ryogo Ema, Seiichiro Sakao, Koichiro Tatsumi 
197. Balloon Pulmonary Angioplasty Improves Biventricular Functions and Pulmonary Flow in Chronic Thromboembolic Pulmonary Hypertension

Haruka Sato, Hideki Ota, Koichiro Sugimura, Tatsuo Aoki, Shunsuke Tatebe, Masanobu Miura, Saori Yamamoto, Nobuhiro Yaoita, Hideaki Suzuki, Kimio Satoh, Kei Takase, Hiroaki Shimokawa

198. Efficacy and Safety of a Novel Endothelin Receptor Antagonist, Macitentan, in Japanese Patients With Pulmonary Arterial Hypertension

Nobuhiro Tahara, Hiroaki Dobashi, Keiichi Fukuda, Masanori Funauchi, Masaru Hatano, Satoshi Ikeda, Shuji Joho, Yasuki Kihara, Takeshi Kimura, Takahisa Kondo, Masakazu Matsushita, Tohru Minamino, Norifumi Nakanishi, Yukio Ozaki, Tsutomu Saji, Satoshi Sakai, Nobuhiro Tanabe, Hiroshi Watanabe, Hidehiro Yamada, Koichiro Yoshioka, Shigetake Sasayama

199. Morphological and Hemodynamic Effectiveness of Stenting for Pulmonary Artery Stenosis - Subanalysis of JPIC Stent Survey -

Takanari Fujii, Hideshi Tomita, Kazuto Fujimoto, Shinichi Otsuki, Toshiki Kobayashi, Yasuo Ono, Satoshi Yazaki, Sung-Hae Kim, Toshio Nakanishi

200. Effects of Proximal Pulmonary Artery Occlusion on Pulsatile Right Ventricular Afterload in Rats Masafumi Fukumitsu, Toru Kawada, Shuji Shimizu, Michael J. Turner, Kazunori Uemura, Masaru Sugimachi

201. Right Heart Hemodynamics in Pulmonary Hypertension - An Echocardiography and Catheterization Study Stéphane Doutreleau, Matthieu Canuet, Irina Enache Paola Di Marco, Evelyne Lonsdorfer, Monique Oswald-Mammoser, Anne Charloux

202. Effects of Balloon Pulmonary Angioplasty on Oxygenation in Patients With Chronic Thromboembolic Pulmonary Hypertension - Importance of Intrapulmonary Shunt -

Tatsuo Aoki, Koichiro Sugimura, Kotaro Nochioka, Masanobu Miura, Shunsuke Tatebe, Saori Yamamoto, Nobuhiro Yaoita, Hideaki Suzuki, Haruka Sato, Katsuya Kozu, Satoshi Miyata, Kimio Satoh, Hiroaki Shimokawa

Regenerative Medicine (1)

203. Angio-Vasculogenic Properties of Endothelial-Induced Mesenchymal Stem Cells Derived From Human Adipose Tissue Long Zhe Guo, Tae-Hee Kim, Seongho Han, Sung-Whan Kim

Renal Disease (5)

204. Levels of Indoxyl Sulfate in Kidney Transplant Patients, and the Relationship With Hard Outcomes Sophie Liabeuf, Lucie Desjardins, Ziad A. Massy, François Brazier, Pierre François Westeel, Hakim Mazouz, Dimitri Titeca-Beauport, Momar Diouf, Griet Glorieux, Raymond Vanholder, Maité Jaureguy, Gabriel Choukroun

205. Early Nephrology Referral 6 Months Before Dialysis Initiation Can Reduce Early Death But Does Not Improve LongTerm Cardiovascular Outcome on Dialysis

Terumasa Hayashi, Tomonori Kimura, Keiko Yasuda, Koichi Sasaki, Yoshitsugu Obi, Harumi Nagayama, Motoki Ohno, Kazusei Uematsu, Takehiro Tamai, Takahiro Nishide, Hiromi Rakugi, Yoshitaka Isaka

206. Combination of Urinary Biomarkers Improves Early Detection of Acute Kidney Injury in Patients With Heart Failure Chia-Hung Yang, Chih-Hsiang Chang, Tien-Hsing Chen, Pei-Chun Fan, Su-Wei Chang, Chun-Chi Chen, Pao-Hsien Chu, Yi-Ting Chen, Huang-Yu Yang, Chih-Wei Yang, Yung-Chang Chen

207. Systemic Inflammation Is Associated With Coronary Artery Calcification and All-Cause Mortality in Chronic Kidney Disease

In-Chang Hwang, Hyo Eun Park, Hack-Lyoung Kim, Hyue Mee Kim, Jun-Bean Park, Yeonyee E. Yoon, Seung-Pyo Lee, Hyung-Kwan Kim, Goo-Yeong Cho, Dae-Won Sohn, Yong-Jin Kim

208. Serum Uric Acid as a Risk Factor for Chronic Kidney Disease in a Japanese Community - The Hisayama Study Keita Takae, Masaharu Nagata, Jun Hata, Naoko Mukai, Yoichiro Hirakawa, Daigo Yoshida, Hiro Kishimoto, Kazuhiko Tsuruya, Takanari Kitazono, Yutaka Kiyohara, Toshiharu Ninomiya

Stroke (5)

209. Greater Severity of Neurological Defects in Women Admitted With Atrial Fibrillation-Related Stroke

Tomohisa Nezu, Naohisa Hosomi, Keita Kondo, Shiro Aoki, Masayasu Matsumoto, Shotai Kobayashi for The Japan Standard Stroke Registry Study Group

210. Statin Adherence After Ischemic Stroke or Transient Ischemic Attack Is Associated With Clinical Outcome Po-Sheng Chen, Ching-Lan Cheng, Yea-Huei Kao Yang, Yi-Heng Li

211. Clinical and Echocardiographic Characteristics of Acute Cardiac Dysfunction Associated With Acute Brain Hemorrhage - Difference From Takotsubo Cardiomyopathy -

Mirae Lee, Ju Hyeon Oh, Kyung Been Lee, Gu Hyun Kang, Yong Hwan Park, Woo Jin Jang, Woo Jung Chun, Sang Hyuk Lee, In Chang Lee

212. Temporal Trends in Stroke Severity and Prior Antithrombotic Use Among Acute Ischemic Stroke Patients in Japan Tomohisa Nezu, Naohisa Hosomi, Gregory YH Lip, Shiro Aoki, Ryo Shimomura, Hirofumi Maruyama, Yoshiki Yagita, Masayasu Matsumoto, Shotai Kobayashi, The Japan Standard Stroke Registry Study Group

213. Human Cytomegalovirus Increases the Risk of Future Hemorrhagic But Not Ischemic Stroke - A Nested Case-Control Study -

Liqiang Zheng, Zhengrong Sun, Zhaoqing Sun, Xingang Zhang, Kai Jing, Jue Li, Dayi Hu, Yingxian Sun 
Valvular Heart Disease (6)

214. Significance of Coronary Artery Disease and Left Ventricular Afterload in Unoperated Asymptomatic Aortic Stenosis Kentaro Shibayama, Masao Daimon, Hiroyuki Watanabe, Takayuki Kawata, Sakiko Miyazaki, Ryoko Morimoto-Ichikawa, Masaki Maruyama, Shuo-Ju Chiang, Katsumi Miyauchi, Hiroyuki Daida

215. Prognostic Significance of ST-Segment Elevation in Leads V $\mathrm{V}_{1-2}$ in Patients With Severe Aortic Stenosis Tomohiko Taniguchi, Hiroki Shiomi, Masami Kosuge, Takeshi Morimoto, Kenji Nakatsuma, Masataka Nishiga, Tomoki Sasa, Naritatsu Saito, Takeshi Kimura

216. Utility of Real-Time 3-Dimensional Transesophageal Echocardiography in the Assessment of Mitral Paravalvular Leak Antonio Arribas-Jimenez, Juan C Rama-Merchan, Manuel Barreiro-Pérez, Soraya Merchan-Gómez, Alberto Iscar-Galán, Ana Martín-García, Felix Nieto-Ballestero, Esther Sánchez-Corral, Javier Rodriguez-Collado, Ignacio Cruz-González, Pedro L Sanchez

217. Predictors of Rapid Progression and Clinical Outcome of Asymptomatic Severe Aortic Stenosis Shunsuke Nishimura, Chisato Izumi, Masataka Nishiga, Masashi Amano, Sari Imamura, Naoaki Onishi, Yodo Tamaki, Soichiro Enomoto, Makoto Miyake, Toshihiro Tamura, Hirokazu Kondo, Kazuaki Kaitani, Yoshihisa Nakagawa

218. Novel Mechanistic Insights Into Atrial Functional Mitral Regurgitation - 3-Dimensional Echocardiographic Study Tomoko Machino-Ohtsuka, Yoshihiro Seo, Tomoko Ishizu, Kimi Sato, Akinori Sugano, Masayoshi Yamamoto, Yoshie Hamada-Harimura, Kazutaka Aonuma

219. Left Atrial Remodeling in Segmental vs. Global Mitral Valve Prolapse - Three-Dimensional Echocardiography Atsushi Hayashi, Shota Fukuda, Keitaro Mahara, Soshi Hei, Takeshi Onoue, Yuichiro Kado, Yasufumi Nagata, Mai Iwataki, Kyoko Otani, Tetsu Miyamoto, Yasushi Oginosawa, Shinjo Sonoda, Masataka Eto, Yosuke Nishimura, Shuichiro Takanashi, Robert A. Levine, Yutaka Otsuji

Vascular Biology and Vascular Medicine (6)

220. Reduction of Intracellular Chloride Concentration Promotes Foam Cell Formation Qian-Qian Wu, Xiao-Yun Liu, Li-Xiong Xiong, Jin-Yan Shang, Xiao-Yi Mai, Rui-Ping Pang, Ying-Xue Su, Bei-Xin Yu, Jia-Ni Yuan, Chao Yang, Yan-Li Wang, Ping Zhou, Xiao-Fei Lv, Jie Liu, Jia-Guo Zhou, Si-Jia Liang

221. Prognostic Implications of Non-Invasive Vascular Function Tests in High-Risk Atherosclerosis Patients Kenya Kusunose, Mitsuyo Sato, Hirotsugu Yamada, Yoshihito Saijo, Mika Bando, Yukina Hirata, Susumu Nishio, Shuji Hayashi, Masataka Sata

222. Molecular Hydrogen Alleviates Cellular Senescence in Endothelial Cells Fumihiko Hara, Junko Tatebe, Ippei Watanabe, Junichi Yamazaki, Takanori Ikeda, Toshisuke Morita

223. Glutamate Promotes Contraction of the Rat Ductus Arteriosus Shujiro Fujita, Utako Yokoyama, Ryo Ishiwata, Rika Aoki, Kenji Nagao, Daiki Masukawa, Masanari Umemura, Takayuki Fujita, Shiho Iwasaki, Shigeru Nishimaki, Kazuo Seki, Shuichi Ito, Yoshio Goshima, Toshihide Asou, Munetaka Masuda, Yoshihiro Ishikawa

224. Endophilin A2 Influences Volume-Regulated Chloride Current by Mediating ClC-3 Trafficking in Vascular Smooth Muscle Cells Can-Zhao Liu, Xiang-Yu Li, Ren-Hong Du, Min Gao, Ming-Ming Ma, Fei-Ya Li, Er-Wen Huang, Hong-Shuo Sun, Guan-Lei Wang, Yong-Yuan Guan

225. Developmental Endothelial Locus-1 (Del-1) Inhibits Oxidized Low-Density Lipoprotein Activity by Direct Binding, and Its Overexpression Attenuates Atherogenesis in Mice

Akemi Kakino, Yoshiko Fujita, Atsushi Nakano, Sayaka Horiuchi, Tatsuya Sawamura

\section{Rapid Communications}

1. Distribution of Anti-Factor Xa Activity in Patients on Edoxaban Therapy for Non-Valvular Atrial Fibrillation Hiroyuki Osanai, Masayoshi Ajioka, Tomohiro Masutomi, Tasuku Kuwayama, Sota Ishihama, Maki Takahashi, Takahiro Kanbara, Yosuke Inoue, Yoshihito Nakashima, Hiroshi Asano, Kazuyoshi Sakai

2. Pulmonary Valve Replacement With Fresh Decellularized Pulmonary Allograft for Pulmonary Regurgitation After Tetralogy of Fallot Repair - First Case Report in Japan -

Takayoshi Ueno, Hideto Ozawa, Masaki Taira, Tomomitsu Kanaya, Koichi Toda, Toru Kuratani, Yoshiki Sawa

3. Increased Coronary Perivascular Adipose Tissue Volume in Patients With Vasospastic Angina Kazuma Ohyama, Yasuharu Matsumoto, Kensuke Nishimiya, Kiyotaka Hao, Ryuji Tsuburaya, Hideki Ota, Hirokazu Amamizu, Hironori Uzuka, Jun Takahashi, Kenta Ito, Hiroaki Shimokawa

4. Feasibility and Safety of Hydrogen Gas Inhalation for Post-Cardiac Arrest Syndrome - First-in-Human Pilot Study Tomoyoshi Tamura, Kei Hayashida, Motoaki Sano, Masaru Suzuki, Takayuki Shibusawa, Joe Yoshizawa, Yosuke Kobayashi, Takeshi Suzuki, Shigeo Ohta, Hiroshi Morisaki, Keiichi Fukuda, Shingo Hori

5. New Stethoscope With Extensible Diaphragm Tsunekazu Takashina, Masashi Shimizu, Torakazu Muratake, Syuichi Mayuzumi

6. Focal Reduction in Cardiac ${ }^{123}$ I-Metaiodobenzylguanidine Uptake in Patients With Anderson-Fabry Disease Saori Yamamoto, Hideaki Suzuki, Koichiro Sugimura, Shunsuke Tatebe, Tatsuo Aoki, Masanobu Miura, Nobuhiro Yaoita, Haruka Sato, Katuya Kozu, Hideki Ota, Kentaro Takanami, Kei Takase, Hiroaki Shimokawa 
7. Early Single Institutional Experience of Berlin Heart EXCOR ${ }^{\circledR}$ Pediatric Ventricular Assist Device in Japan

Takashi Kakuta, Takaya Hoashi, Heima Sakaguchi, Koji Kagisaki, Jun Negishi, Masatoshi Shimada, Etsuko Tsuda, Isao Shiraishi, Norihide Fukushima, Hajime Ichikawa

Images in Cardiovascular Medicine

1. Cor Triatriatum Sinister - Source of Unusual Thrombogenesis in Mitral Stenosis Chan-Hee Lee, Jang-Won Son, Dong-Gu Shin

2. Successful Management of Guidewire Kinking in a Patient With Subaortic Septal Bulging Using the Pull-Through Technique During Transapical Transcatheter Aortic Valve Implantation

Toshiaki Toyota, Naritatsu Saito, Kenji Minakata, Masao Imai, Kyokun Uehara, Hiroomi Nishio, Yuki Kuroda, Hirotoshi Watanabe, Tomohiko Taniguchi, Junichi Tazaki, Kazuhiro Yamazaki, Hiroki Daijo, Takeshi Kimura

3. Coronary Stenosis as an Innocent Bystander in Acute Coronary Syndrome Italo Porto, Domenico D’Amario, Lazzaro Paraggio, Filippo Crea

4. Cardiac Arrest Triggered by Subepicardial Aneurysm Without Cardiac Rupture Eriko Hasumi, Katsuhito Fujiu, Teruhiko Imamura, Hiroshi Iwata, Daigo Sawaki, Yumiko Hosoya, Jiro Ando, Toshiya Kojima, Yu Shimizu, Gaku Oguri, Takumi Matsubara, Masaru Hatano, Hiroshi Akazawa, Masafumi Watanabe, Minoru Ono, Issei Komuro

5. Rapid Pacing-Induced Massive Mitral Regurgitation During Transcatheter Aortic Valve Implantation Juan Caro-Codón, Silvia Valbuena-López, Carlos Álvarez-Ortega, Mar Moreno-Yangüela, Raúl Moreno

6. Reversible Microvascular Dysfunction in Takotsubo Syndrome Shown Using Index of Microcirculatory Resistance Takayuki Warisawa, Toru Naganuma, Sunao Nakamura

7. Recurrent Aortitis Associated With IgG4-Related Disease Antonio Lalueza, Sebastián Ruiz, Felipe Villacampa, Rocío López, Elena Zabia, Pilar Sarandeses, Ana Cristina Hernández, Juan María Herrero, Carlos Lumbreras

8. Steroid Therapy Ameliorated Myocardial Fatty Acid Metabolism With Recovery of Complete Atrioventricular Block in Cardiac Sarcoidosis

Takashige Tobita, Mitsuru Momose, Atsushi Suzuki, Kenji Fukushima, Chisato Kondo, Kenta Uto, Koichiro Abe, Tsuyoshi Shiga, Shuji Sakai, Nobuhisa Hagiwara

9. Severely Calcified and Dilated Meandering Mesenteric Artery Complicating Abdominal Aorta Stenosis Takayasu Ito, Ryuji Okamoto, Takashi Tanigawa, Tatsuya Kawaguchi, Hideo Mizutani, Eitaro Fujii, Norikazu Yamada, Masaaki Ito

10. Intraprocedural Stent Thrombosis Detected on Coronary Angioscopy Toru Miyoshi, Hideo Kawakami, Akira Oshita, Hiroshi Matsuoka

11. Erdheim-Chester Disease With Cardiovascular Involvement and BRAF V600E Mutation Kenichi Okamura, Yoshihiro Suematsu, Sei Morizumi, Mitsuhiro Kawata, Yuichi Dai, Mitsunori Yamakawa, Minoru Ono

12. Partial Anomalous Pulmonary Venous Connection With Left Atrial Drainage of the Superior Vena Cava Tai Fuchigami, Nobuhiro Nagata, Masahiko Nishioka, Toru Akashige, Shotaro Higa, Mami Nakayashiro, Taisuke Nabeshima, Kazuhiro Takahashi

13. Endomyocardial Fibrosis Associated With Apical Calcification and High Uptake on Myocardial Gallium-67 Scintigraphy Sonoko Maemura, Eisuke Amiya, Hikari Seki, Kazutaka Ueda, Daisuke Nitta, Teruhiko Imamura, Masae Uehara, Takayuki Kawata, Masafumi Watanabe, Masaru Hatano, Koichiro Kinugawa, Issei Komuro

14. Yellow Neointima Following Stent Implantation in the Superficial Femoral Artery on Angioscopy Koji Yanaka, Takayuki Ishihara, Osamu Iida, Shin Okamoto, Masashi Fujita, Masaharu Masuda, Kiyonori Nanto, Tatsuya Shiraki, Takashi Kanda, Masaaki Uematsu

15. Focal Vasa Vasorum Formation in Patients With Focal Coronary Vasospasm - An Optical Frequency Domain Imaging Study -

Kensuke Nishimiya, Yasuharu Matsumoto, Hironori Uzuka, Kazuma Ohyama, Kiyotaka Hao, Ryuji Tsuburaya, Takashi Shiroto, Jun Takahashi, Kenta Ito, Hiroaki Shimokawa

16. Tumor Plop Sound Ryota Kakizaki, Takeru Nabeta, Tomohiro Mizutani, Toshimi Koitabashi, Junya Ako

17. Purulent Pericarditis Accompanying Pericardial Abscess Induced by Nocardia in an Immunocompromised Patient Akira Takashima, Shusuke Yagi, Koji Yamaguchi, Syunsuke Watanabe, Nobuaki Yamamoto, Hiroyuki Ito, Muneyuki Kadota, Tomoya Hara, Hiromu Yamazaki, Takayuki Ise, Kenya Kusunose, Takeshi Tobiume, Hirotsugu Yamada, Takeshi Soeki, Tetsuzo Wakatsuki, Masataka Sata

18. Morphological Assessment of Single-Ventricle Atrioventricular Valve Regurgitation on Dual-Source 128-Slice Multidetector Computed Tomography and 4-Dimensional Imaging Yoshihiko Kodama, Makoto Nakamura, Koichi Sagawa, Shiro Ishikawa, Toshihide Nakano, Hideaki Kado

19. Unexpected Atrial Septal Intramural Hematoma During Coronary Angiography Michiaki Kono, Yoritaka Otsuka, Masaaki Kawahara, Yuki Imoto, Taku Koyama, Keita Nakamura, Sunao Kodama, Hiroo Noguchi 


\section{JCS Guidelines}

1. Guidelines for Disaster Medicine for Patients With Cardiovascular Diseases (JCS 2014/JSH 2014/JCC 2014) - Digest Version -

JCS, JSH and JCC Joint Working Group

2. Guidelines for Diagnosis and Treatment of Patients With Hypertrophic Cardiomyopathy (JCS 2012) - Digest Version JCS Joint Working Group

\section{Letters to the Editor}

1. Does Age Have an Effect on Systemic Inflammatory Response? (Letter) Orhan Gokalp, Ali Gurbuz, Nihan Karakas Yesilkaya, Yuksel Besir, Gamze Gokalp (Reply) Suguru Ohira, Hitoshi Yaku

2. Screening of Coronary Artery Disease in Diabetic Patients: Who and How? (Letter) Jesus M de la Hera, Maria Martín, Cecilia Corros, Ana García-Campos, Juan Calvo (Reply) Michio Shimabukuro, Taro Saito, Hiroaki Masuzaki, Masataka Sata

3. Effects of Primary Aortic Disease on Patient-Prosthetic Valve Mismatch (Letter) Orhan Gokalp, Bortecin Eygi, Yuksel Besir, Gamze Gokalp, Ali Gurbuz (Reply) Kenji Minakata, Ryuzo Sakata

4. Ischemic Lesion Formation in Solitary Tract Nuclei During Central Sleep Apnea With Heart Failure

(Letter) J. Howard Jaster

(Reply) Shin-ichi Momomura

........ 1047

(Reply) Koichiro Sugimura, Hiroyuki Satake, Hiroaki Shimokawa

….... 1048

….... 1049

5. Balloon Atrial Septostomy in Congenital Heart Disease (Letter) Arun Gopalakrishnan, Bijulal Sasidharan, Kavassery Mahadevan Krishnamoorthy (Reply) Hisashi Sugiyama, Kazuto Fujimoto, Tetsuko Ishii, Toshio Nakanishi, Hideshi Tomita

6. Intraoperative Factors Cause Postoperative Atrial Fibrillation? (Letter) Orhan Gokalp, Bortecin Eygi, Yuksel Besir, Gamze Gokalp, Ali Gurbuz (Reply) Shinya Takahashi, Taijiro Sueda

7. Respiratory Therapy in Chronic Heart Failure Patients Complicated With Sleep-Disordered Breathing: Potential Study Bias

(Letter) Monica Felix-Moscoso, Jack Denegri-Galvan, Fernando Ortega-Loayza, Adrian V. Hernandez

….... 1486 (Reply) Koichiro Sugimura, Hiroyuki Satake, Hiroaki Shimokawa

8. Point-of-Care Device for Warfarin Monitoring Used in the J-ROCKET AF Study (Letter) On behalf of the J-ROCKET AF Study Investigators, Masatsugu Hori, Yohei Ohashi, Guohua Pan, Masaharu Kato, Mariko Kajikawa

9. Is T1-Mapping Truly Superior to Late Gadolinium Enhancement-Imaging in Demonstrating Myocardial Fibrosis in Myopathy- and Non-Myopathy Associated Noncompaction?

(Letter) Josef Finsterer, Claudia Stöllberger

(Reply) Hongmei Zhou, Xue Lin, Ligang Fang, Haiyan Ding, Quan Fang

10. Treatment of Spontaneous Isolated Superior Mesenteric Artery Dissection (Letter) Zhongzhi Jia, Shaoqin Li, Guomin Jiang

(Reply) Koichi Tomita, Hideaki Obara, Kentaro Matsubara, Naoki Fujimura, Yuko Kitagawa

……. 1876

11. Has the Safety of Edoxaban $60 \mathrm{mg}$ Among East Asian Atrial Fibrillation Patients Been Truly Proven by the ENGAGE AF-TIMI 48 Subanalysis? (Letter) Tomiya Yasumasu (Reply) Takeshi Yamashita

12. Documentation of Focal Takotsubo Syndrome and Its Diagnostic Implications (Letter) John E. Madias

(Reply) Ken Kato, Yoshio Kobayashi

13. Effect of Using Bilateral Internal Mammary Artery on Long-Term Survival (Letter) Orhan Gokalp, Yuksel Besir, Hasan Iner, Levent Yilik, Gamze Gokalp, Ali Gurbuz (Reply) Satoshi Itoh, Naoyuki Kimura, Hideo Adachi, Atsushi Yamaguchi

14. Predictors of Hemodynamically Successful Stenting for Pulmonary Artery Stenosis (Letter) Tomoaki Murakami (Reply) Takanari Fujii, Hideshi Tomita

15. Pulmonary Vascular Resistance in Patients With Pulmonary Hypertension: Importance of the Quadratic Velocity-Pressure Relationship (Letter) Amr El Sayed Abbas

16. Management of Gene-Positive Catecholaminergic Polymorphic Ventricular Tachycardia: Are the Long Term Outcomes on Therapy Really So Poor?

(Letter) Thomas M. Roston, Shubhayan Sanatani 